OPEN ACCESS

Check for updates

\title{
Efficacy of covid-19 vaccines in immunocompromised patients: systematic review and meta-analysis
}

\author{
Ainsley Ryan Yan Bin Lee, ${ }^{1}$ Shi Yin Wong, ${ }^{1}$ Louis Yi Ann Chai,,${ }^{2,3,4,5}$ Soo Chin Lee, ${ }^{6,7}$ \\ Matilda Xinwei Lee, ${ }^{6}$ Mark Dhinesh Muthiah, ${ }^{8,9}$ Sen Hee Tay, ${ }^{10}$ Chong Boon Teo, ${ }^{1}$ \\ Benjamin Kye Jyn Tan, ${ }^{1}$ Yiong Huak Chan, ${ }^{11}$ Raghav Sundar, ${ }^{3,6,12,13,14}$ Yu Yang Soon ${ }^{3,15}$
}

For numbered affiliations see end of the article

Correspondence to: R Sundar mdcragh@nus.edu.sg

(or@sundar_raghav on Twitter; ORCID 0000-0001-9423-1368) Additional material is published online only. To view please visit the journal online.

Cite this as: BMJ 2022;376:e068632 http://dx.doi.org/10.1136/ bmj-2021-068632

Accepted: 24 January 2022

\section{ABSTRACT}

OBJECTIVE

To compare the efficacy of covid-19 vaccines between immunocompromised and immunocompetent people.

\section{DESIGN}

Systematic review and meta-analysis.

\section{DATA SOURCES}

PubMed, Embase, Central Register of Controlled Trials, COVID-19 Open Research Dataset Challenge (CORD-19), and WHO covid-19 databases for studies published between 1 December 2020 and 5 November 2021. ClinicalTrials.gov and the WHO International Clinical Trials Registry Platform were searched in November 2021 to identify registered but as yet unpublished or ongoing studies.

\section{STUDY SELECTION}

Prospective observational studies comparing the efficacy of covid-19 vaccination in immunocompromised and immunocompetent participants.

\section{METHODS}

A frequentist random effects meta-analysis was used to separately pool relative and absolute risks of seroconversion after the first and second doses of a covid-19 vaccine. Systematic review without meta-analysis of SARS-CoV-2 antibody titre levels was performed after first, second, and third vaccine doses and the seroconversion rate after a third dose. Risk of bias and certainty of evidence were assessed.

RESULTS

82 studies were included in the meta-analysis. Of these studies, 77 (94\%) used mRNA vaccines, 16 (20\%) viral vector vaccines, and 4 (5\%) inactivated

\section{WHAT IS ALREADY KNOWN ON THIS TOPIC \\ Immunocompromised patients show lower seroconversion rates than immunocompetent people after vaccination, such as with the influenza vaccine Less is known about the response to covid-19 vaccines, particularly mRNA based vaccines}

\section{WHAT THIS STUDY ADDS}

Seroconversion rates after covid-19 vaccination were found to be reduced among all immunocompromised groups, except people with HIV, but significantly increased after the second dose; in organ transplant recipients seroconversion remained severely reduced even after a second dose

Among the immunocompromised groups studied, antibody titres were lower than in immunocompetent controls

These findings suggest that a third dose of covid-19 vaccine would be efficacious in immunocompromised patients whole virus vaccines. 63 studies were assessed to be at low risk of bias and 19 at moderate risk of bias. After one vaccine dose, seroconversion was about half as likely in patients with haematological cancers (risk ratio $0.40,95 \%$ confidence interval 0.32 to $0.50,1^{2}=80 \%$; absolute risk 0.29 , $95 \%$ confidence interval 0.20 to $0.40, \mathrm{I}^{2}=89 \%$ ), immune mediated inflammatory disorders $\left(0.53,0.39\right.$ to $0.71, \mathrm{I}^{2}=89 \%$; $0.29,0.11$ to $\left.0.58, \mathrm{I}^{2}=97 \%\right)$, and solid cancers $(0.55$, 0.46 to $0.65, \mathrm{I}^{2}=78 \% ; 0.44,0.36$ to $0.53, \mathrm{I}^{2}=84 \%$ ) compared with immunocompetent controls, whereas organ transplant recipients were 16 times less likely to seroconvert $\left(0.06,0.04\right.$ to $0.09, \mathrm{I}^{2}=0 \% ; 0.06,0.04$ to $\left.0.08, l^{2}=0 \%\right)$. After a second dose, seroconversion remained least likely in transplant recipients $(0.39$, 0.32 to $0.46, I^{2}=92 \% ; 0.35,0.26$ to 0.46$)$, with only a third achieving seroconversion. Seroconversion was increasingly likely in patients with haematological cancers $\left(0.63,0.57\right.$ to $0.69, \mathrm{I}^{2}=88 \% ; 0.62,0.54$ to $\left.0.70, \mathrm{I}^{2}=90 \%\right)$, immune mediated inflammatory disorders $\left(0.75,0.69\right.$ to $0.82, \mathrm{I}^{2}=92 \% ; 0.77,0.66$ to $\left.0.85, \mathrm{I}^{2}=93 \%\right)$, and solid cancers $(0.90,0.88$ to $0.93, \mathrm{I}^{2}=51 \% ; 0.89,0.86$ to $0.91, \mathrm{I}^{2}=49 \%$ ). Seroconversion was similar between people with HIV and immunocompetent controls $(1.00,0.98$ to 1.01 , $\mathrm{I}^{2}=0 \% ; 0.97,0.83$ to $\left.1.00, \mathrm{I}^{2}=89 \%\right)$. Systematic review of 11 studies showed that a third dose of a covid-19 mRNA vaccine was associated with seroconversion among vaccine non-responders with solid cancers, haematological cancers, and immune mediated inflammatory disorders, although response was variable in transplant recipients and inadequately studied in people with HIV and those receiving nonmRNA vaccines.

\section{CONCLUSION}

Seroconversion rates after covid-19 vaccination were significantly lower in immunocompromised patients, especially organ transplant recipients. A second dose was associated with consistently improved seroconversion across all patient groups, albeit at a lower magnitude for organ transplant recipients. Targeted interventions for immunocompromised patients, including a third (booster) dose, should be performed.

\section{SYSTEMATIC REVIEW REGISTRATION} PROSPERO CRD42021272088.

\section{Introduction}

Transmission of SARS-CoV-2 has led to the ongoing global covid-19 pandemic. By November 2021, more than 250 million have had confirmed covid-19 and more than four million have died worldwide. 
The morbidity and mortality from covid-19 and its complications and large scale economic disruption have prompted an unprecedented pace in vaccine development. ${ }^{12}$ Vaccines that have been approved for use to date include new technology mRNA vaccines such as BNT162b2 (Pfizer-BioNTech) and mRNA1273 (Moderna), non-replicating viral vector vaccines such as Janssen's Ad26.COV2.S (Johnson \& Johnson), and traditional inactivated whole virus vaccines such as CoronaVac (Sinovac Biotech). ${ }^{3}$ Trials and ongoing studies have sought to evaluate the efficacy and safety of these vaccines. High vaccine efficacy against symptomatic laboratory confirmed SARSCoV-2 infection has been reported, with more than $50 \%$ efficacy after the first dose of BNT162b2 and 90\% after the second dose, ${ }^{4}$ whereas Oxford-AstraZeneca reported an efficacy of $70 \%$ after the second dose of its viral vector vaccine, AZD1222 (ChAdOx1 nCoV-19). ${ }^{5}$ High seroconversion rates were shown regardless of class of vaccine used or previous infection status. ${ }^{6}$

Vaccine trials, however, have excluded immunocompromised groups, such as patients with cancer, organ transplant recipients, and those with rheumatological disorders, leading to a paucity of data on the efficacy and safety of vaccines in these groups. These patients, who comprise about 3\% of the adult population, ${ }^{7}$ are of particular interest because of possible suppression or over-activation of the immune system attributable to the primary disease or concurrent treatment. Data are urgently needed on immunocompromised patients, as infection and viral shedding have been reported to be more severe and persistent in this group. ${ }^{89}$ Patients with active cancer are known to be at increased risk of severe covid-19 and death. ${ }^{10} 11$ Transplant recipients require prolonged immunosuppression to reduce the risk of graft rejection, and past studies have shown increased risk of severe covid-19 and poor outcomes. ${ }^{12}$ Patients requiring immunosuppressive treatment for autoimmune and inflammatory rheumatic diseases have worse outcomes from covid-19 than age and sex comparable patients without such conditions. ${ }^{13}$ People with HIV are also at higher risk of hospital admission for severe covid-19, and in-hospital mortality. ${ }^{14}$

Studies have shown variable efficacy of other vaccines, such as influenza and pneumococcal vaccines, among immunocompromised groups depending on factors such as vaccine type, underlying disease, and concurrent drugs. In a meta-analysis on the immunogenicity of influenza vaccination in organ transplant recipients, the risk factors for lower seroconversion included transplantation within six months, receiving antimetabolites, and lung transplantation. ${ }^{15}$ Other studies have shown reduced antibody response after the influenza vaccine among patients with cancer, organ transplant recipients, and those receiving other anti-CD20 based immunosuppressive drugs, such as rituximab in those with rheumatic conditions. ${ }^{1617}$

To date, no systematic reviews have been performed on the immunogenicity of covid-19 vaccines in immunocompromised cohorts. In this review we compared seroconversion rates and antibody titre levels for different covid-19 vaccines between immunocompromised patients and immunocompetent controls.

\section{Methods}

This systematic review is reported according to the Preferred Reporting Items for Systematic Reviews and Meta-Analyses (PRISMA) guidelines. ${ }^{18}$

\section{Search strategy}

In November 2021 and according to the study protocol, we searched several databases: Medline via PubMed, Embase, Cochrane Central Register of Controlled Trials (CENTRAL), CORD-19, WHO COVID-19 Research Database, ClinicalTrials.gov, and WHO international clinical trials registry platform for articles published from 1 December 2020 to 5 November 2021 (see supplementary table 1 for search strategy). No restrictions on language of publication were applied. To improve validity of data, we excluded non-peer reviewed articles in preprint databases.

\section{Study selection}

We used a two stage approach for screening: first by title and abstract and then by full text article. Two researchers (ARYBL and SYW) independently screened each title, abstract, and full text, with discrepancies resolved by consensus with a third researcher. Studies were limited to human participants and of any followup duration and timepoints.

We performed meta-analysis of prospective observational studies that met several criteria: included human participants who received a covid-19 vaccine of any brand and type; included patients with active cancer of solid organs; patients with active haematological cancers; organ transplant recipients; patients with active immune mediated inflammatory disorders ${ }^{19}$ except asthma or receiving immunosuppressive or immunomodulatory drugs; people with HIV/AIDS; studies that included and reported data on a control group comprising immunocompetent people or comparator groups of people who were not immunocompromised, defined as not having cancer, immune mediated inflammatory disorders, organ transplant conditions, or HIV; and studies that reported at least one of seroconversion after covid-19 vaccination or serological titres after covid-19 vaccination.

We excluded studies that included but did not report outcomes of an immunocompetent control group; reported seroconversion data in a form that prevented the calculation of proportions, risk of seroconversion, or number of seroconverted participants; and reported serological titres in a form from which neither mean nor median titres could be derived.

When studies did not have available data, we emailed the corresponding authors for information. We excluded studies only if data were not provided at the time of meta-analysis. 
Given the emergence of clinical studies of a third dose of covid-19 vaccine in immunocompromised patients, we made a post hoc amendment to include studies reporting these data, for qualitative analysis: prospective observational or experimental studies that involved human participants, all of whom should be receiving a covid-19 vaccine of any brand and type; studies that involved patients with solid organ cancer, haematological cancer, immune mediated inflammatory disorders, HIV/AIDS, and organ transplant recipients; and studies that reported seroconversion rates among immunocompromised patients, with or without the inclusion of an immunocompetent control group.

\section{Data extraction}

Two researchers (ARYBL and SYW) extracted data according to a predetermined proforma in Microsoft Excel Version 16.45. All key extracted data were reviewed and quality checked at the end of the data extraction phase by the same two researchers.

Data on study characteristics comprised setting, primary and secondary outcomes, study design, sample size, dropout and non-response rates, and inclusion and exclusion criteria. Participant data comprised age, sex, and disease and treatment history, including immunosuppressive regimen. Intervention related data included vaccine type and brand, dosing schedule, number of participants receiving each type and brand of vaccine, and median or mean interval between doses. Outcome related data comprised assay type, antibody measured, method of measurement, intervals of sample collection, and number of measurements.

\section{Risk of bias assessment}

The Risk Of Bias In Non-randomized Studies of Interventions (ROBINS-I) tool was used to rate risk of bias for non-randomised included studies. This tool assesses seven domains: risk of bias from confounding, selection of participants, classification of interventions, deviations from intended interventions, missing data, measurement of outcomes, and selection of the reported results. ${ }^{20}$ Two reviewers (ARYBL and SYW) independently judged these domains as having low, moderate, serious, or critical risk of bias, or no information. All discrepancies were resolved by the independent opinion of a third reviewer. A study would be judged as having an overall low risk of bias if all the domains were judged as low risk. A study would be considered as having critical risk of bias if one domain was judged as high risk of bias.

The Cochrane Risk of Bias 2.0 tool was to be used for randomised trials. During our search, however, no eligible randomised studies were found. ${ }^{21}$

\section{Outcomes of interest}

The primary outcomes of interest were seroconversion after a first and second dose of covid-19 vaccine. As brand and type of assay, type of immunoglobulin, and definition of seroconversion differed across studies, supplementary table 2 reports the respective data from each study. We sought direct evidence of vaccine protection, including asymptomatic and symptomatic covid-19 infection, need for oxygen supplementation, and hospital or intensive care unit stay in both immunocompromised patients and immunocompetent controls. No studies met our inclusion criteria for these outcomes.

Secondary outcomes of interest were mean or median serological titres and cumulative incidence of seroconversion after a first and second dose of covid-19 vaccines. As the type of antibodies measured and reported differed across studies, supplementary tables 3 and 4 show the titres after a first and second vaccine dose, respectively. Supplementary table 2 shows the timepoints of serological assessment after covid-19 vaccination and the different brands of serological kits.

\section{Data analysis}

We used the DerSimonian and Laird random effects model to estimate the pooled risk ratios and corresponding 95\% confidence intervals for the primary outcomes of interest. A risk ratio $<1$ indicates that the immunocompromised participants had a lower risk of achieving seroconversion after covid-19 vaccination compared with immunocompetent controls. Statistical heterogeneity of the results in the included studies was assessed by $\chi^{2}$ test and $\mathrm{I}^{2}$ statistic. We considered heterogeneity to be significant when the P value by $\chi^{2}$ test was $<0.10$, or the $\mathrm{I}^{2}$ statistic was $\geq 50 \%$. ${ }^{22}$

Assessment for publication bias was both qualitative, through visual inspection for funnel plot asymmetry, and quantitative, using Egger's test. We performed subgroup analyses to determine if results were influenced by the type of haematological cancers, solid cancers, immune mediated diseases, organ transplants, and covid-19 vaccines. Interaction tests were used to compare the differences between estimates from different subgroups.

As we did not expect the covid-19 antibody titre levels to be amenable to statistical pooling because of different methods and assays used, secondary outcomes were assessed using the synthesis without meta-analysis approach.

We performed separate meta-analyses for the relative risk of seroconversion (measured as risk ratios compared with immunocompetent controls) and the absolute risk of seroconversion (measured as a proportion from $0-100 \%$ ) after each vaccine dose. Generalised linear mixed effects models were used to pool the logit transformed proportions of immunocompromised patients who achieved seroconversion after a first and second covid-19 vaccine dose. Between study heterogeneity was considered significant if the $P$ value of Cochran's $Q$ test was $<0.10$, or if the $\mathrm{I}^{2}$ statistic was $\geq 50 \%$. ${ }^{22}$

Small study effects were assessed both qualitatively, through visual inspection for funnel plot asymmetry, and quantitatively, using Egger's test. We conducted all analyses on $\mathrm{R}$ (version 4.0.3) using the meta and metafor packages. Unless specified otherwise, we considered a two sided $\mathrm{P}$ value of $<0.05$ to be statistically significant. 
When publication bias was suspected based on either the Egger's regression-intercept test of bias or visual inspection for funnel plot asymmetry, we conducted a sensitivity analysis using the trim-and-fill method (R0 estimator, fixed random effects models) to re-estimate the pooled effect size after imputing potentially missing studies. ${ }^{23} 24$ The trim-and-fill method shows a normal distribution of effect sizes around the centre of the funnel plot if publication bias is absent. $^{25}$

\section{Certainty of evidence}

We assessed the certainty of evidence using the Grading of Recommendations Assessment, Development, and Evaluation (GRADE). ${ }^{26}$ Certainty of the overall evidence was rated as high, moderate, low, or very low based on the assessment of the domains for risk of bias, inconsistency, indirectness, publication bias, intransitivity, incoherence, and imprecision.

\section{Patient and public involvement}

No patients or members of the public were directly involved in this research study.

\section{Results}

Figure 1 shows the selection of studies. Overall, 82 studies were included for meta-analysis (table 1, supplementary table 2). ${ }^{27-108}$ Supplementary tables 3 and 4 present the serological antibody titres after a first and second dose of covid-19 vaccines, respectively. Five studies were included for qualitative analysis of titres but not for meta-analysis of seroconversion rates. ${ }^{109-113}$ In addition, 11 studies reporting seroconversion data of a third vaccine dose were included for qualitative analysis (see supplementary table 5). ${ }^{43114-123}$ One study was included in both meta-analysis and qualitative analysis of a third vaccine dose. ${ }^{43}$ Five studies that met the inclusion criteria for meta-analysis were excluded because missing data could not be obtained in time from the corresponding authors. Four studies did not report seroconversion rates among immunocompetent controls. One study included patients with cancer and organ transplant recipients, but did not provide seroconversion data for each group separately.

Of the 82 included studies, 77 (94\%) used mRNA vaccines BNT162b2 (Pfizer-BioNTech) and mRNA1273 (Moderna), with 66 (80\%) studies involving only mRNA vaccines, $16(20 \%)$ viral vector vaccines, and four $(5 \%)$ inactivated vaccines (CoronaVac, Sinovac, Biotech). Among the viral vector vaccines, AZD1222 (ChAdOx1 nCoV-19; Oxford-AstraZeneca) featured more prominently, being used in $16(20 \%)$ studies but as the sole vaccine in only one (1\%). Ad26.COV2.S (Janssen/Johnson \& Johnson) was used in only one study (in 3/289 immunocompetent controls and no immunocompromised patients). ${ }^{29}$

Trials primarily included immunocompromised groups of organ transplant recipients and patients with cancers and immune mediated inflammatory disorders. Eighteen (22\%) studies included patients with solid cancers, 21 (26\%) included patients with haematological cancer, 17 (20.7\%) included patients with immune mediated inflammatory disorders, 26 (32\%) included organ transplant recipients, and four (5\%) included people with HIV.

\section{First vaccine dose}

Figure 2 presents the risk ratios for seroconversion among immunocompromised patients compared with immunocompetent controls after a first dose of covid-19 vaccine.

Thirty five studies reported seroconversion after a first vaccine dose in immunocompromised patients $(n=4052)$ compared with immunocompetent controls $(n=3856)$. See supplementary tables 2 and 3 for details of the studies.

Among the immunocompromised groups, risk ratios were lowest for organ transplant recipients, with minimal heterogeneity (risk ratio 0.06, 95\% confidence interval 0.04 to $0.09, \mathrm{I}^{2}=0 \%$ ) (moderate certainty of evidence), followed by patients with haematological cancers $\left(0.40,0.32\right.$ to $\left.0.50, \mathrm{I}^{2}=80 \%\right)$ (moderate certainty of evidence), immune mediated inflammatory disorders $\left(0.53,0.39\right.$ to $\left.0.71, \mathrm{I}^{2}=89 \%\right)$ (moderate certainty of evidence), and solid cancers $\left(0.55,0.46\right.$ to $\left.0.65, \mathrm{I}^{2}=78 \%\right)$ (moderate certainty of evidence). Only one study reported the seroconversion rate in people with HIV and found no difference compared with immunocompetent controls (1.06, 0.74 to 1.54 ) (low certainty of evidence) ${ }^{.10} 3$

\section{Second vaccine dose}

Figure 3 and figure 4 show the risk ratios for seroconversion among immunocompromised patients compared with immunocompetent controls after a second dose of covid-19 vaccine. See supplementary tables 2 and 4 for details of the studies.

A total of 73 studies including 77 immunocompromised cohorts reported seroconversion in 9974 immunocompromised patients compared with 6215 immunocompetent controls after a second vaccine dose.

Among the immunocompromised groups, the lowest pooled risk ratio after a second vaccine dose was observed in organ transplant recipients $(0.39$, 0.32 to $0.46, \mathrm{I}^{2}=92 \%$ ) (moderate certainty evidence), followed by patients with haematological cancers (0.63, 0.57 to $0.69, \mathrm{I}^{2}=88 \%$ ) (low certainty evidence), immune mediated inflammatory disorders $(0.75,0.69$ to $0.82, \mathrm{I}^{2}=92 \%$ ) (low certainty evidence), and solid cancers $\left(0.90,0.88\right.$ to $0.93, \mathrm{I}^{2}=51 \%$ ) (low certainty of evidence), and people with HIV (1.00, 0.98 to 1.01 , $\mathrm{I}^{2}=0 \%$ ) (low certainty of evidence).

\section{Vaccine response}

\section{Patients with solid cancers: first dose}

Eleven studies including 827 patients with solid cancers and 1653 immunocompetent controls showed a pooled risk ratio of 0.55 (0.46 to 0.65 ) for seroconversion after a first dose of covid-19 vaccine (fig 2). See supplementary tables 2 and 3 for details of the studies. 


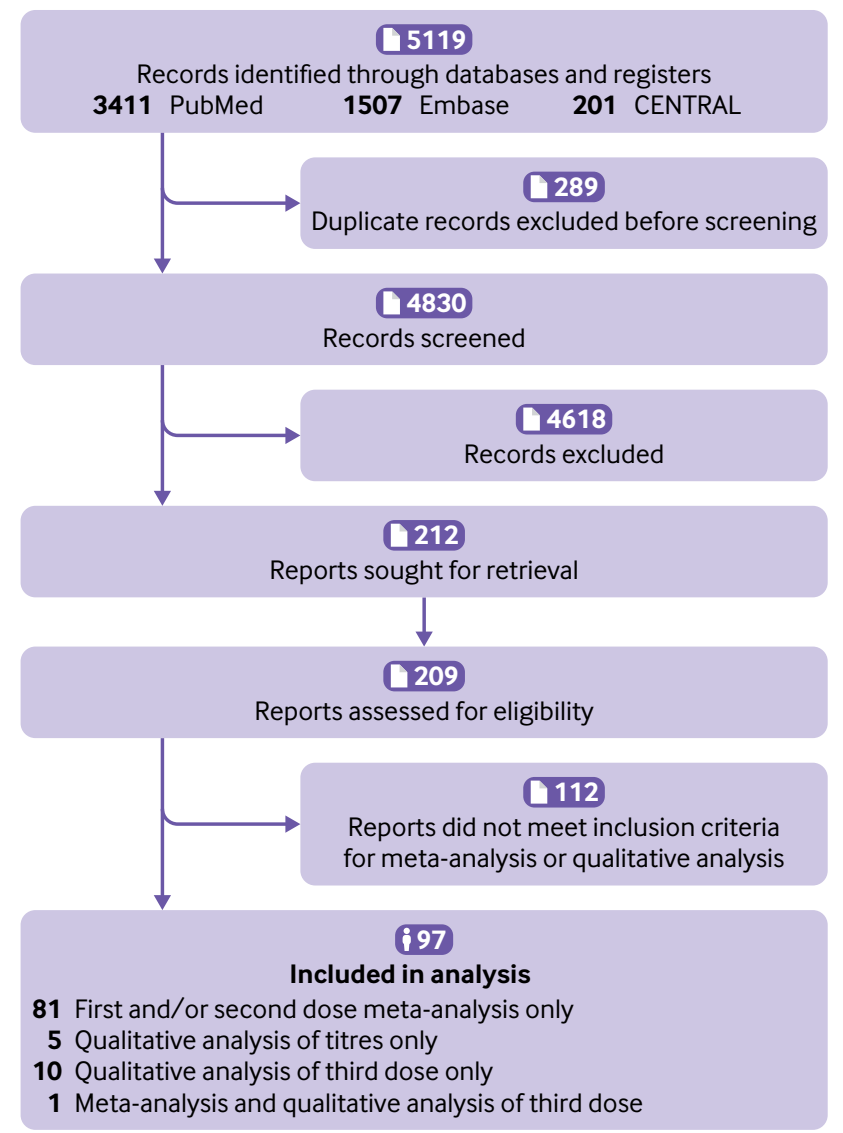

Fig 1 | Flowchart of study selection

Barriere et $\mathrm{al}^{108}$ showed the most significant reduction in antibody titres, of 41.5 -fold, among 122 immunocompromised patients (median 0.52 $\mathrm{UI} / \mathrm{mL}$, range 0-1962 UI/mL) compared with 13 immunocompetent controls $(21.6 \mathrm{UI} / \mathrm{mL}$, range 3.26-723.2 UI/mL), whereas Zagouri et $\mathrm{al}^{44}$ showed the least significant reduction, of 1.08 -fold, among 18 immunocompromised patients (median 39.5\%) compared with 160 immunocompetent controls (median 42.83\%). Liontos et al found lower antibody titres among immunocompetent controls compared with immunocompromised patients. The difference was not statistically significant $(\mathrm{P}=0.26) .^{38}$

\section{Patients with solid cancers: second dose}

Fourteen studies including 1488 patients with solid cancers and 1607 immunocompetent controls showed a pooled risk ratio of 0.90 (0.88 to 0.93$)$ for seroconversion after a second vaccine dose (fig 3 ). See supplementary tables 2 and 4 for details of the studies.

Barriere et al found the most significant reduction in antibody titres, of 10.3-fold, among 42 immunocompromised patients (median 245.2 $\mathrm{UI} / \mathrm{mL}$, range 0-5467 UI/mL) compared with 24 immunocompetent controls (median $2517 \mathrm{UI} / \mathrm{mL}$, range 157.6-6318.0 UI/mL). ${ }^{108}$ Liontos et al, however, found comparable neutralising antibody titres among patients with breast and prostate cancer compared with immunocompetent controls. ${ }^{37} 38$
A reduction in seroconversion rates was found in patients with solid cancer after both vaccine doses, with a significant increase of 1.64 times between the first and second dose (pooled risk ratio 0.55, 0.46 to 0.65 and $0.90,0.88$ to 0.93 , respectively).

\section{Patients with haematological cancers: first dose} Eleven studies including 1123 patients with haematological cancers and 1346 immunocompetent controls showed a pooled risk ratio of 0.40 (0.32 to 0.50) for seroconversion after a first dose of covid-19 vaccine (fig 2). See supplementary tables 2 and 3 for details of the studies.

Lim et al reported the most significant reduction in antibody titres, of 935.6-fold and 79.6-fold among immunocompromised patients (geometric mean titre 2.5 binding antibody units (BAU)/mL, 95\% confidence interval 1.1 to $5.8 \mathrm{BAU} / \mathrm{mL}$ ) compared with 65 and 20 immunocompetent controls who received BNT162b2 (2339 BAU/mL, 40 to $111 \mathrm{BAU} / \mathrm{mL}$ ) and AZD1222 (199 BAU/mL, 140 to $282 \mathrm{BAU} / \mathrm{mL}$ ). ${ }^{59}$ Pimpinelli et al, however, reported less of a reduction in antibody titres, of 2.28-fold (median 7.5 arbitrary units (AU)/mL, 95\% confidence interval 5.6 to $10.4 \mathrm{AU} / \mathrm{mL}$ ) among patients with multiple myeloma and of 1.06-fold (median 16.2 $\mathrm{AU} / \mathrm{mL}, 11.7$ to $22.3 \mathrm{AU} / \mathrm{mL}$ ) among patients with myeloproliferative neoplasm. ${ }^{46}$

Patients with haematological cancers: second dose Nineteen studies including 2436 patients with haematological cancers and 1896 immunocompetent controls showed a pooled risk ratio of $0.63(95 \%$ confidence interval 0.57 to 0.69 ) for seroconversion after a second dose of covid-19 vaccine (fig 3). See supplementary tables 2 and 4 for details of the studies.

Herishanu et $\mathrm{al}^{45}$ found the most significant reduction, of 1315.5 -fold, among 52 patients with chronic lymphocytic leukaemia (median $0.824 \mathrm{U} / \mathrm{mL}$, interquartile range $0.4-167.3 \mathrm{U} / \mathrm{mL}$ ) compared with 52 immunocompetent controls (median $1084 \mathrm{U} / \mathrm{mL}$, interquartile range 128.9-1879 U/mL) after the second vaccine dose. The smallest reduction was reported in the study by Gavriatopoulou et al, of 1.85-fold among 90 patients with haematological cancers (mean $52 \%$, interquartile range 47.7-56.3\%) compared with 108 immunocompetent controls (mean $87.4 \%$, interquartile range $86.1-88.7 \%) .^{52}$

Seroconversion rates in patients with haematological cancers were reduced after both vaccine doses, with a significant increase of 1.58 -fold between the first and second dose (pooled risk ratio $0.40,95 \%$ confidence interval 0.32 to 0.50 and $0.63,0.57$ to 0.69 , respectively).

\section{Patients with immune mediated inflammatory disorders: first dose}

Seven studies reported seroconversion rates among patients with immune mediated inflammatory disorders after a first dose of covid-19 vaccine (fig 2). Data from 1526 patients compared with 602 immunocompetent controls showed that the patients 


\begin{tabular}{|c|c|}
\hline Characteristics & No $(\%)(n=82)$ \\
\hline \multicolumn{2}{|l|}{ Morbidity* } \\
\hline Solid cancers & $18(22)$ \\
\hline Haematological cancers & $21(26)$ \\
\hline Immune mediated inflammatory disorders & $17(21)$ \\
\hline Organ transplants & $26(32)$ \\
\hline HIV/AIDS & $4(5)$ \\
\hline \multicolumn{2}{|l|}{ Vaccine dose } \\
\hline First & $35(43)$ \\
\hline Second & $73(89)$ \\
\hline \multicolumn{2}{|l|}{ Country } \\
\hline Israel & $18(22)$ \\
\hline Germany & $12(15)$ \\
\hline USA & $11(13)$ \\
\hline Greece & $9(11)$ \\
\hline UK & $7(9)$ \\
\hline France & $7(9)$ \\
\hline Italy & $5(6)$ \\
\hline Belgium & $2(2)$ \\
\hline Brazil & $2(2)$ \\
\hline Switzerland & $2(2)$ \\
\hline South Africa & $1(1)$ \\
\hline Netherlands & $1(1)$ \\
\hline Austria & $1(1)$ \\
\hline Spain & $1(1)$ \\
\hline Thailand & $1(1)$ \\
\hline Turkey & $1(1)$ \\
\hline Poland & $1(1)$ \\
\hline \multicolumn{2}{|l|}{ Vaccine types } \\
\hline \multicolumn{2}{|l|}{ mRNA: } \\
\hline BNT162b2 (Pfizer-BioNTech) and mRNA-1273 (Moderna) & 77 (94); only mRNA vaccines in 66 (80) \\
\hline \multicolumn{2}{|l|}{ Non-replicating viral vector: } \\
\hline AZD1222 (ChAdOx1 nCoV-19; Oxford-AstraZeneca) & 16 (20); sole vaccine in 1 (1) \\
\hline Ad26.COV2.S (Janssen/Johnson \& Johnson)† & $1(1)$ \\
\hline \multicolumn{2}{|l|}{ Inactivated: } \\
\hline CoronaVac (Sinovac Biotech) & $4(5)$ \\
\hline \multicolumn{2}{|c|}{$\begin{array}{l}\text { At the time of the studies, recommended vaccine regimens included two vaccine doses, except for Ad26.COV2.S } \\
\text { (Janssen/Johnson \& Johnson), which only required one dose. The current meta-analysis further stratified results } \\
\text { according to seroconversion and antibody titres after a first dose and second dose. } \\
{ }^{*} \text { Study by Rahav et } a^{27} \text { included patients with solid cancers, haematological cancers, immune mediated } \\
\text { inflammatory disorders, organ transplants, and HIV/AIDS. Study by Monin et } a^{28}{ }^{28} \text { included patients with solid } \\
\text { cancers and haematological cancers. } \\
\text { †Vaccine used in only } 3 / 289(1 \%) \text { immunocompetent controls in study by Boekel et a' } 29\end{array}$} \\
\hline
\end{tabular}

had a pooled risk ratio of 0.53 (95\% confidence interval 0.39 to 0.71 ). See supplementary tables 2 and 3 for details of the studies.

Lower antibody titres were seen after a first vaccine dose among patients with immune mediated inflammatory disorders. Rubbert-Roth et al found that the antibody titres of 51 patients with rheumatoid arthritis (median $0.4 \mathrm{U} / \mathrm{mL}$, interquartile range 0.4-2.13 U/mL) were much lower than those of 20 immunocompetent controls $(99.2 \mathrm{U} / \mathrm{mL}, 24.8-172 \mathrm{U} /$ $\mathrm{mL}$ ), of 248 -fold. ${ }^{89}$ Medeiros-Ribeiro et al showed a less significant decrease in 859 patients with immune mediated inflammatory disorders (median $5.1 \mathrm{AU} / \mathrm{mL}$, interquartile range 4.7-5.5 AU/mL) compared with 179 immunocompetent controls (10.3 AU/mL, 8.5-12.5 $\mathrm{AU} / \mathrm{mL}$ ), of 2.02-fold. ${ }^{93}$

\section{Patients with immune mediated inflammatory} disorders: second dose

Seventeen studies also reported seroconversion rates among patients with immune mediated inflammatory disorders after a second dose of covid-19 vaccine (fig
4). Data from 2668 patients compared with 1296 immunocompetent controls showed that the patients had a pooled risk ratio of 0.75 ( $95 \%$ confidence interval 0.69 to 0.82 ). See supplementary tables 2 and 4 for details of the studies.

Achiron et al reported the greatest and smallest reduction in antibody titres among patients with immune mediated inflammatory disorders, which provided insights into the impact of individual immunosuppressive agents on antibody titres in patients with multiple sclerosis within a median range of 2.3-6.3 months after a second vaccine dose. ${ }^{99}$ The greatest reductions in antibody titres were seen at 23.7fold, 35.5-fold, and 8.88-fold for the 42 patients taking fingolimod (median 0.3, 95\% confidence interval 0.3 to 0.7$), 114$ patients taking ocrelizumab $(0.2,0.6$ to $1.2)$, and six patients taking rituximab (0.8, 0.7 to 5.2$)$ compared with 89 immunocompetent controls (7.1, 6.2 to 7.1 ). The smallest reductions were 1.01-fold among 22 patients taking alemtuzumab (7.0, 5.7 to 8.3), 1.08-fold among 48 patients taking cladribine (6.6, 5.7 to 6.8), 0.93-fold among 35 patients taking dimethyl fumarate (7.6, 7.1 to 7.9), 0.97-fold among 32 patients taking natalizumab (7.3, 6.6 to 7.8$)$, and 1.04fold among 39 patients taking teriflunomide $(6.8,6.1$ to 7.2) compared with the same 89 immunocompetent controls.

Seroconversion rates in patients with immune mediated inflammatory disorders were reduced after both doses, with a significant increase of 1.42 times between the first and second dose (pooled risk ratio $0.53,95 \%$ confidence interval 0.39 to 0.71 and 0.75 , 0.69 to 0.82 , respectively).

\section{Organ transplant recipients: first dose}

Six studies reported seroconversion in 540 organ transplant recipients compared with 266 immunocompetent controls after a first dose of covid-19 vaccine (fig 2). The reduction in seroconversion rates was significant (pooled risk ratio 0.06, 0.04 to 0.09) and largely homogenous between studies $\left(\mathrm{I}^{2}=0 \%\right)$. See supplementary tables 2 and 3 for details of the studies.

Canti et al reported the most significant reduction in antibody titres, of 64.2-fold among 37 transplant recipients (median $6 \mathrm{IU} / \mathrm{mL}$, interquartile range 2.5$77.5 \mathrm{IU} / \mathrm{mL}$ ) compared with 40 immunocompetent controls (median $385.4 \mathrm{IU} / \mathrm{mL}$, interquartile range 148.2-554.7 IU/mL) after the first dose.

\section{Organ transplant recipients: second dose}

Across 24 studies, when data were pooled for 3051 transplant organ recipients and 1679 immunocompetent controls, a strong risk for nonseroconversion was observed, with the lowest pooled risk ratio being 0.39 (0.32 to 0.46$)$. See supplementary tables 2 and 4 for details of the studies.

Narasimhan et al reported the greatest difference in antibody titres between organ transplant recipients and immunocompetent controls: $1.7 \mathrm{AU} / \mathrm{mL}$ (95\% confidence interval 0.6 to $7.5 \mathrm{AU} / \mathrm{mL}$ ) and 14209 $\mathrm{AU} / \mathrm{mL}$ (11261 to $18836 \mathrm{AU} / \mathrm{mL}$ ), respectively, with 


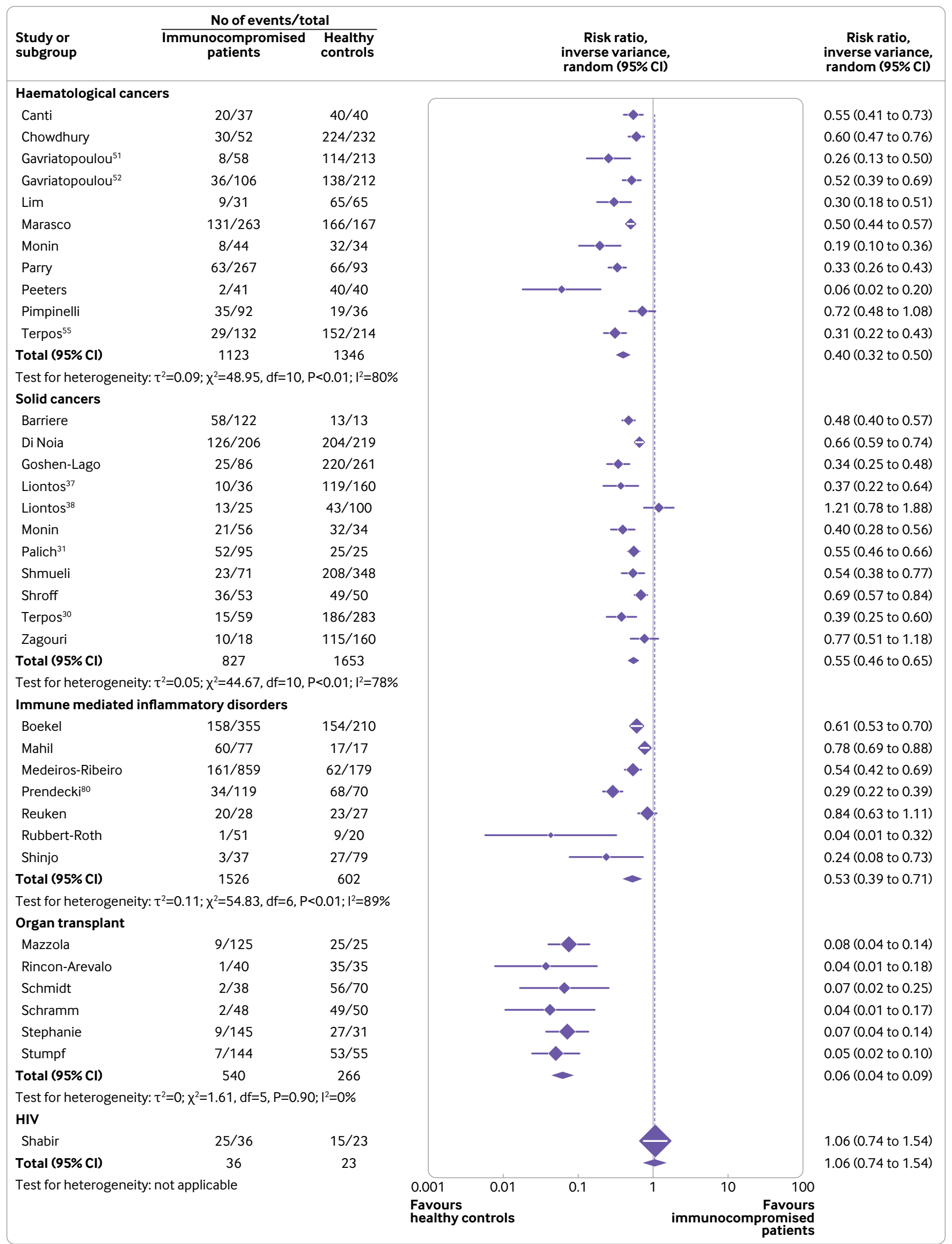

Fig 2 | Risk ratios for seroconversion among immunocompromised patients compared with immunocompetent controls after a first dose of covid-19 vaccine 


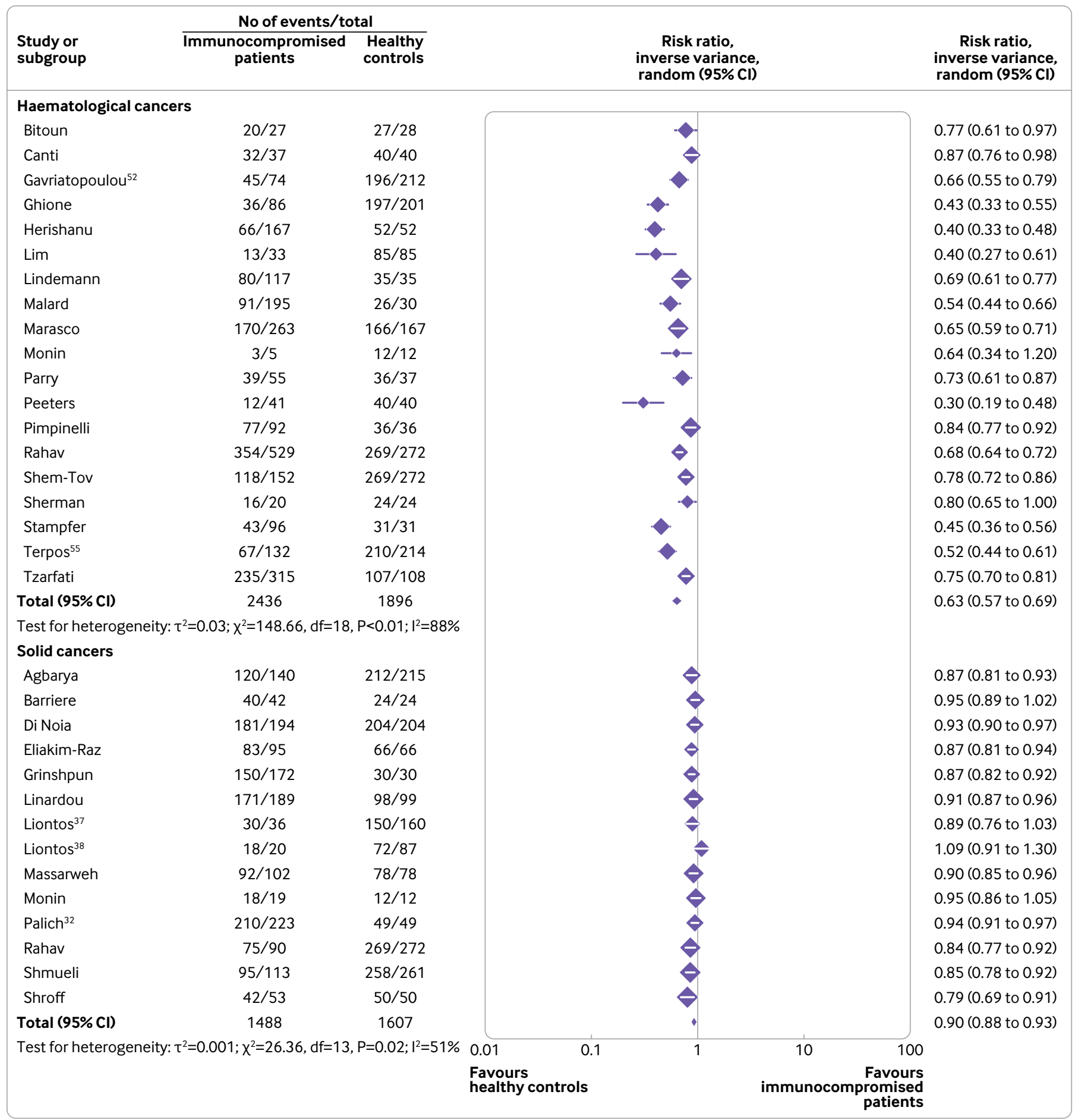

Fig 3 | Risk ratios for seroconversion among immunocompromised patients with haematological cancers and solid cancers compared with immunocompetent controls after a second dose of covid-19 vaccine

a difference of $8358.24 \mathrm{AU} / \mathrm{mL}^{73}$ Rabinowich et al reported the smallest reduction in antibody titres, of 2.10-fold (mean 95.41 (standard deviation 92.4) AU/ $\mathrm{mL} v 200.5$ (65.1) AU/mL). ${ }^{66}$

Seroconversion rates in organ transplant recipients were reduced after both vaccine doses, with a significant increase of 6.5 -fold between the first and second dose (pooled risk ratio 0.06, 0.04 to 0.09 and $0.39,0.32$ to 0.46 , respectively).
These results suggest that a second dose of covid-19 vaccine is imperative in improving seroconversion rates in organ transplant recipients. Seroconversion rates remained reduced compared with immunocompetent controls, however, necessitating future study for third (booster) doses for such patients. Non-vaccine protective measures would also be vital in protecting this vulnerable patient group. 




Fig 4 | Risk ratios for seroconversion among immunocompromised patients with immune mediated inflammatory disorders, organ transplant recipients, and people with HIV compared with immunocompetent controls after a second dose of covid-19 vaccine 
People with HIV: first and second doses

In one study with 36 people with HIV and 23 immunocompetent controls, seroconversion rates were comparable among the two populations (risk ratio $1.06,0.74$ to 1.54$)$ after the first dose of covid-19 vaccine. See supplementary table 2 for details of the study.

In four studies, with a total of 331 people with HIV and 565 immunocompetent controls, seroconversion rates were high among the people with HIV (pooled risk ratio $1.00,0.98$ to 1.01) after a second dose of covid-19 vaccine (fig 4). See supplementary tables 2 and 4 for details of the studies.

Antibody titres did not seem to be significantly affected or reduced among participants after a second vaccine dose, with Rahav et $\mathrm{al}^{27}$ reporting 1.16-fold reduction among 88 people with HIV (median 18.7 relative units (RU)/mL, interquartile range 0-42 RU/mL) compared with 41 immunocompetent controls (median $20 \mathrm{RU} / \mathrm{mL}$, interquartile range 12-27 RU/mL) and Jedicke et $\mathrm{al}^{112}$ reporting a 0.74 -fold reduction among 26 controls (median $26 \mathrm{RU} / \mathrm{mL}$, interquartile range 18$37 \mathrm{RU} / \mathrm{mL}$ ) compared with 52 people with HIV (median $35 \mathrm{RU} / \mathrm{mL}$, interquartile range 1-128 RU/mL).

\section{Heterogeneity in immunocompromised patients after two doses}

Subgroup analysis was performed according to study and patient level categorical characteristics to account for heterogeneity in seroconversion observed after the first and second doses of covid-19 vaccine (see supplementary tables 6-11). Among studies of patients with haematological cancer, subgroup analysis was performed of studies that included only patients with one type of haematological cancer (see supplementary figure 1 and supplementary table 9). This analysis was performed in patients with multiple myeloma ( $\mathrm{n}=2$ studies; risk ratio $0.59,95 \%$ confidence interval 0.35 to 0.99 ), recipients of haematopoietic stem cell transplant ( $\mathrm{n}=5 ; 0.75,0.68$ to 0.83$)$, and patients with chronic lymphocytic leukaemia $(\mathrm{n}=2 ; 0.54,0.30$ to 0.98). In the subgroup analyses among patients with haematological cancer, a significant subgroup effect was found according to type of cancer $(\mathrm{P}=0.01$ for test of subgroup effect).

A similar subgroup analysis was performed among studies of patients with immune mediated inflammatory disorders (see supplementary figure 2 and supplementary table 10). This analysis included patients with inflammatory bowel diseases $(n=2$ studies; risk ratio $0.97,0.89$ to 1.07 ), multiple sclerosis ( $\mathrm{n}=3 ; 0.50,0.35$ to 0.70$)$, and rheumatoid arthritis ( $\mathrm{n}=1 ; 0.83,0.73$ to 0.94$)$. In subgroup analyses among patients with immune mediated inflammatory disorders, a significant subgroup effect was found according to type of disease $(\mathrm{P}<0.001$ for test of subgroup effect).

Subgroup analysis of organ transplant recipients showed that seroconversion differed depending on the organ transplanted (see supplementary figure 3 and supplementary table 11). In increasing order of risk ratios, these were heart $(\mathrm{n}=2$ studies; 0.16 , 0.11 to 0.25 ), lung ( $\mathrm{n}=1 ; 0.25,0.17$ to 0.37 ), kidney $(\mathrm{n}=11 ; 0.34,0.26$ to 0.45$)$, and liver $(\mathrm{n}=4 ; 0.66,0.55$ to 0.80 ). The remaining six studies involving patients with various solid organ transplants $(n=6 ; 0.41,0.34$ to 0.50 ) showed significantly reduced heterogeneity. Overall, the subgroup effect was significant $(\mathrm{P}<0.001$ for test of subgroup effect).

Subgroup analysis was performed for studies involving only mRNA vaccines and only non-mRNA vaccines (see supplementary figure 4). Owing to the limited number of studies of only non-mRNA vaccines, subgroup analysis was only possible for patients with immune mediated inflammatory disorders after the second dose (see supplementary table 10). No significant differences $(\mathrm{P}=0.65$ for test of subgroup effect) were found in effects on seroconversion between mRNA vaccines (risk ratio $0.72,0.64$ to 0.81 ) and nonmRNA vaccines $(0.78,0.69$ to 0.88$)$.

In subgroup analysis, the brand of serology kit and country of study were of inconsistent significance across immunocompromised groups, and hence are unlikely to be a major confounder overall (see supplementary tables 6-11). Significant subgroup differences for brand of serology kit were observed in patients with immune mediated inflammatory disorders and organ transplant recipients after the second vaccine dose and patients with haematological cancer after both the first and the second dose. Similarly, subgroups by country of study yielded significant differences only in patients with immune mediated inflammatory disorders and organ transplant recipients after the second dose and patients with solid cancer after both the first and the second dose.

Mixed effects metaregression of seroconversion against potential effect moderators (continuous and categorical study level characteristics), including average age of patients, brand of serology kit for assays, timepoints for assays after covid-19 vaccination, and risk of bias of study, did not show any consistent effect moderation across immunocompromised populations after both the first or the second dose (see supplementary tables 12-13 and supplementary tables 14-17, respectively).

All studies included in this meta-analysis involved immunocompetent controls to improve comparability of data. Responses in immunocompetent controls were homogenous across studies. Heterogeneity in metaanalysis of seroconversion risk in immunocompromised patients was largely in accordance with that of seroconversion risk ratios (see supplementary tables 18-19 and supplementary figures 5-13).

\section{Risk of bias assessment}

Sixty three studies were assessed to be at low risk of bias and 19 at moderate risk of bias (see supplementary table 20). No studies were considered at severe or critical risk of bias. Risk of bias was mainly related to confounding effects, with controls not being age matched, or missing data, with recruited patients lacking available data at predetermined endpoints. 
Publication bias and trim-and-fill analysis for patients with cancer: first and second dose

Supplementary figures 14 to 21 show the funnel plot with trim-and-fill imputation of potentially missing studies for organ transplant recipients and patients with solid cancer, haematological cancer, and immune mediated inflammatory disorders after first and second doses. Publication bias was absent for most analyses, except for some concern in those involving organ transplant recipients and patients with haematological cancer after the second dose.

\section{Immunogenicity of a third covid-19 vaccine dose}

Ten prospective observational studies and one randomised controlled trial reported data after a third dose of covid-19 vaccine. Most of the studies (8/11 studies) were on organ transplant recipients, ${ }^{115-122}$ with one study each on patients with immune mediated inflammatory disorders, ${ }^{123}$ haematological cancers, ${ }^{114}$ and solid cancers. ${ }^{43}$ Nine studies utilised only mRNA vaccines (mRNA-1273 or BNT162b2), whereas two studies $^{114} 121$ administered either BNT162b2 or AZD1222 as a third dose after two doses of BNT162b2.

Most studies did not report data for controls and hence did not meet the inclusion criteria for the metaanalysis. Given that the efficacy of the third dose is an emerging area of global interest, data are summarised in supplementary table 5 .

Systematic review without meta-analysis showed that a third dose of a covid-19 mRNA vaccine dose might improve seroconversion, with rates in organ transplant recipients mostly between $36.0 \%$ and $66.7 \%$. Only one study, by Chavarot et al, reported significantly impaired seroconversion, at the lowest rate of $6.5 \%$. Response was highly variable in organ transplant recipients. ${ }^{115}$ No published evidence was available on the efficacy of a third dose in people with HIV.

\section{Discussion}

In this systematic review and meta-analysis of 82 studies, we found that immunocompromised groups of organ transplant recipients and patients with solid cancers, haematological cancers, and immune mediated inflammatory disorders had depressed seroconversion after a first and second dose of covid-19 vaccine compared with immunocompetent controls. Compared with immunocompetent controls, the pooled risk ratios for seroconversion after the first vaccine dose were lower among organ transplant recipients (risk ratio 0.06) and patients with haematological cancers (0.40), immune mediated inflammatory disorders (0.53), and solid cancers (0.55). Antibody response improved significantly after the second dose. The pooled risk ratios after the second dose increased to 0.39 among organ transplant recipients, 0.63 among patients with haematological cancers, 0.75 among patients with immune mediated inflammatory disorders, and 0.90 among patients with solid cancers. Although the number of studies were insufficient for meta-analysis of seroconversion after the first dose among people with HIV, the immune response to covid-19 vaccines was shown to be preserved after the second dose (risk ratio 1.00). Organ transplant recipients showed sustained low seroconversion rates after both vaccine doses.

Our findings highlight the importance of the second dose of covid-19 vaccines and subsequent third vaccine (booster) doses. The benefits of additional doses and boosters of vaccines are well established, both for covid-19 ${ }^{124-126}$ and for pre-existing vaccines, such as the inactivated polio vaccine. ${ }^{127}$ This review similarly highlights the importance of a second dose of covid-19 vaccine, especially for immunocompromised patients. Across the included studies, a second dose of vaccine was associated with greatly improved seroconversion and antibody titre levels. In particular, a second dose was associated with increasing immunogenicity and protection in organ transplant recipients and patients with haematological cancers.

\section{Benefits of a third dose of covid-19 vaccine}

In organ transplant recipients and patients with haematological cancers, our results showed a less than ideal seroconversion rate, even after a second vaccine dose, prompting the need for additional measures. Particularly, seroconversion rates were severely reduced in organ transplant recipients across all studies.

Del Bello et al showed increasing seroconversion with each dose (from one to three) of the BNT162b2 vaccine (Pfizer-BioNTech) in organ transplant recipients. ${ }^{117}$

In August 2021, the US Food and Drug Administration authorised the use of a third dose of Pfizer-BioNTech and Moderna vaccines for immunocompromised populations, including organ transplant recipients, ${ }^{128}$ with other countries following suit. ${ }^{129}$

Our meta-analyses suggest significant heterogeneity in immunogenicity between different immunocompromised groups, after both the first and the second dose of covid-19 vaccines. The response noticeably varied in organ transplant recipients after the second dose. This might be attributed to changes in immunosuppressive regimens in the transplant populations after emergence of data on poor responses to a first vaccine dose, or the release of a multi-society joint statement advocating vaccination for all organ transplant recipients midway through several of the reported studies. ${ }^{10} 130$ Vaccine regimes may need to be tailored according to the cause and degree of immunocompromise. One of the included studies, by Achiron et al, also found significantly different seroconversion rates for patients receiving different treatments. ${ }^{99}$ This finding is orthogonally supported by a study from Kennedy et al, which underscored the fact that immunosuppression caused by different biological agents could be substantial; 20 patients receiving infliximab had significantly lowered titres compared with seven patients receiving vedolizumab (mean 158 (SD 7.0) U/mL $v 562$ (SD 11.5) U/mL). ${ }^{131}$ Ligumsky et $\mathrm{al}^{132}$ further found that different 
anticancer treatments are also associated with varying seroconversion rates and antibody titres, with patients who receive chemotherapy showing a lower median IgG titre and seroconversion rate than those receiving immune checkpoint inhibitors and targeted treatment.

Surrogate measures of immunogenicity and efficacy Currently, no international consensus exists on measures to determine immunogenicity. Trials reported surrogate measures, including seroconversion rates and geometric mean titres. These surrogate measures involved parameters related to anti-SARSCOV-2 recombinant spike, receptor binding domain, or neutralising IgG or total antibodies. The use of immunological markers in predicting protection against covid-19 has been the subject of much debate. ${ }^{133-136}$ Although the neutralising antibody level has more recently been established as a reliable predictor of protection against symptomatic covid-19, the measures used in many studies varied.

In this review, only studies that compared measures of effect between immunocompromised patients and immunocompetent controls were included.

\section{Understudied populations}

Minimal published data are currently available that compare the immunogenicity of covid-19 vaccines between people with HIV/AIDS and immunocompetent people. Studies have established lower rates of response to hepatitis $B$ and influenza vaccines in people with HIV/AIDS, making study of covid-19 vaccines in this immunocompromised population pertinent. ${ }^{17137-139}$

Our meta-analysis shows that the seroconversion rate in people with HIV was comparable to that in immunocompetent controls. Given the HIV/AIDS spectrum, the effect of level of depletion of CD4 counts on immune response remains to be established.

Data are also lacking in patients with primary immunodeficiencies. ${ }^{140}$ However, even patients with primary antibody deficiencies such as combined variable immunodeficiency have been shown to develop anti-spike antibodies after covid-19 vaccination. ${ }^{141}$ All patients with primary immunodeficiencies therefore should be vaccinated against covid-19.

\section{Efficacy of mRNA vaccines versus non-mRNA vaccines}

The covid-19 mRNA vaccines represent a new class of vaccine products. It is difficult to directly compare the seroconversion rates of the covid-19 mRNA vaccines with more traditional, frequently used vaccines. In our study, we found no significant differences in a subgroup analysis of mRNA and conventional vaccines in patients with immune mediated inflammatory disorders. The study by Fan et al, which compared mRNA vaccines with conventional vaccines in nonimmunocompromised patients, ${ }^{142}$ summarised the safety and efficacy of the three main vaccine platforms (mRNA, non-replicating viral vector, inactivated) reported in phase III trials. When patients with confirmed covid-19 were compared over the total number of participants in each (vaccinated $v$ control) group, mRNA vaccines appeared to be the most efficacious after two doses (risk ratio 0.05, 95\% confidence interval 0.02 to 0.13 ) compared with nonreplicating viral vector vaccines $(0.33,0.22$ to 0.50$)$ and inactivated vaccines $(0.32,0.23$ to 0.42$)$. A similar trend could be observed after one dose.

A review suggests that the immune response to the influenza vaccine might not be as strong in immunocompromised patients, yet they appear to derive some benefit from vaccination. ${ }^{143}$ These findings reflect what is now being experienced with covid-19 and vaccination.

\section{Limitations of this study}

This study has several limitations. Firstly, the included studies are observational. Factors that might influence the immune response to the vaccine, such as comorbidities and age, might not be controlled for between the immunocompromised group and immunocompetent control group. To deal with this limitation, we performed subgroup analyses, which showed no significant effect modification between studies with participants of different median age.

Secondly, the definition of immunocompromised varied between studies. We therefore prespecified the definition of immunocompromised and performed subgroup analyses to assess the difference in seroconversion rates in groups of immunocompromised patients. These analyses showed noticeable differences between organ transplant recipients, people with HIV, and patients with solid cancers, haematological cancers, and immune mediated inflammatory disorders.

Although the seroconversion rate is an indication of an immune response to a vaccine, it is only a proxy for the effects of the vaccine on infection rates and severity of covid-19. Data are still lacking on clinical efficacy endpoints such as covid-19 infection rates in vaccinated immunocompromised populations.

Lastly, the definition of seroconversion and the type of immunoassay used were not standardised across the studies. To deal with this limitation, we performed subgroup analyses to determine the presence of effect modification between studies that used different brands of immunoassays. The findings were inconsistent. Furthermore, vaccine type might influence seroconversion rates after covid-19 vaccination. Given that the studies included in this review predominantly used mRNA vaccines, however, analyses of possible differences were limited.

\section{Conclusion}

Inthismeta-analysis, wehaveshownthatseroconversion rates and antibody titres after covid-19 vaccines are significantly lower in immunocompromised patients than immunocompetent individuals. Among the various groups of immunocompromised patients, organ transplant recipients showed the lowest rates of seroconversion, whereas patients with solid cancers showed the highest. Notably, immunocompromised 
patients generally develop lower antibody titres with seroconversion than immunocompetent controls, which raises concern about the adequacy of seroprotection. Additional strategies, such as the administration of a third vaccine dose to the conventional two dose regimen for mRNA covid-19 vaccines would be warranted to confer improved seroprotection for these patients.

\section{AUTHOR AFFILIATIONS}

${ }^{1}$ Yong Loo Lin School of Medicine, National University of Singapore, Singapore

${ }^{2}$ Division of Infectious Diseases, Department of Medicine, National University Health System, Singapore

${ }^{3}$ Department of Medicine, Yong Loo Lin School of Medicine, National University of Singapore, Singapore

${ }^{4}$ Synthetic Biology for Clinical and Technological Innovation, National University of Singapore, Singapore

${ }^{5}$ National University Cancer Institute, Singapore

${ }^{6}$ Department of Haematology-Oncology, National University Cancer Institute, National University Hospital, 119228, Singapore

${ }^{7}$ Cancer Science Institute of Singapore, National University of Singapore, Singapore

${ }^{8}$ Department of Gastroenterology and Hepatology, National University Health System, Singapore

${ }^{9}$ National University Centre for Organ Transplantation, Singapore

${ }^{10}$ Division of Rheumatology, Department of Medicine, National University Hospital, Singapore

${ }^{11}$ Biostatistics Unit, Yong Loo Lin School of Medicine, National University of Singapore, Singapore

${ }^{12}$ Cancer and Stem Cell Biology Program, Duke-NUS Medical School, Singapore

${ }^{13}$ The N.1 Institute for Health, National University of Singapore, Singapore

${ }^{14}$ Singapore Gastric Cancer Consortium, Singapore.

${ }^{15}$ Department of Radiation Oncology, National University Cancer Institute, Singapore

Contributors: ARYBL and SYW contributed equally to this paper and are joint first authors. RS and YYS contributed equally to this paper and are joint last authors. ARYBL, SYW, RS, and YYS conceived and designed the study. ARYBL and SYW selected the articles and extracted data. BKJT, CBT, YHC, ARYBL, and YYS were responsible for statistical analysis. ARYBL and SYW wrote the first draft of the manuscript. RS, YYS, LYAC, SCL, MDM, SHT, and ML provided advice at different stages. All authors approved the final version of the manuscript. RS is the guarantor. The corresponding author attests that all listed authors meet authorship criteria and that no others meeting the criteria have been omitted.

Funding: This study did not receive specific funding. RS is supported by the National Medical Research Council (NMRC/MOH/000627).

Competing interests: All authors have completed the ICMJE uniform disclosure form at www.icmje.org/coi disclosure.pdf and declare: no support from any organisation for the submitted work; no financial relationships with any organisations that might have an interest in the submitted work in the previous three years; no other relationships or activities that could appear to have influenced the submitted work. Ethical approval. Not required.

Data sharing: No additional data available.

The lead author (RS) affirms that the manuscript is an honest, accurate, and transparent account of the study being reported; that no important aspects of the study have been omitted; and that any discrepancies from the study as planned have been explained.

Dissemination to participants and related patient and public communities: We will disseminate the results to clinicians, patients, and governmental organisations and agencies through social media. Provenance and peer review: Not commissioned; externally peer reviewed.

This is an Open Access article distributed in accordance with the Creative Commons Attribution Non Commercial (CC BY-NC 4.0) license, which permits others to distribute, remix, adapt, build upon this work non-commercially, and license their derivative works on different terms, provided the original work is properly cited and the use is non commercial. See: http://creativecommons.org/licenses/by-nc/4.0/.

1 Berlin DA, Gulick RM, Martinez FJ. Severe Covid-19. N Engl Med 2020;383:2451-60. doi:10.1056/NEJMcp2009575

2 Ali MAM, Spinler SA. COVID-19 and thrombosis: From bench to bedside. Trends Cardiovasc Med 2021;31:143-60. doi:10.1016/j. tcm.2020.12.004

3 Fathizadeh H, Afshar S, Masoudi MR, et al. SARS-CoV-2 (Covid-19) vaccines structure, mechanisms and effectiveness: A review. Int I Biol Macromol 2021;188:740-50. doi:10.1016/j.ijbiomac.2021.08.076

4 Polack FP, Thomas SJ, Kitchin N, et al, C4591001 Clinical Trial Group. Safety and Efficacy of the BNT162b2 mRNA Covid-19 Vaccine. N Engl J Med 2020:383:2603-15. doi:10.1056/NEJMoa2034577

5 Voysey M, Clemens SAC, Madhi SA, et al, Oxford COVID Vaccine Trial Group. Safety and efficacy of the ChAdOx1 nCoV-19 vaccine (AZD1222) against SARS-CoV-2: an interim analysis of four randomised controlled trials in Brazil, South Africa, and the UK Lancet 2021;397:99-111. doi:10.1016/S0140-6736(20)32661-1

6 Eyre DW, Lumley SF, Wei I, et al. Quantitative SARS-CoV-2 anti-spike responses to Pfizer-BioNTech and Oxford-AstraZeneca vaccines by previous infection status. Clin Microbiol Infect 2021;27:1516.e7-14. doi:10.1016/j.cmi.2021.05.041

7 Centers for Disease Control and Prevention. Covid-19 vaccines for moderately to severely immunocompromised people. CDC. [Cited 27 September 2021]. www.cdc.gov/coronavirus/2019-ncov/vaccines/ recommendations/immuno.html

8 Manuel O, Estabrook M, American Society of Transplantation Infectious Diseases Community of Practice. RNA respiratory viral infections in solid organ transplant recipients: Guidelines from the American Society of Transplantation Infectious Diseases Community of Practice. Clin Transplant 2019;33:e13511. doi:10.1111/ctr.13511

9 Couch RB, Englund JA, Whimbey E. Respiratory viral infections in immunocompetent and immunocompromised persons. Am I Med 1997;102(3A):2-9, discussion 25-6. doi:10.1016/S0002 9343(97)00003-X

10 Mohammed AH, Blebil A, Dujaili J, Rasool-Hassan BA. The Risk and Impact of COVID-19 Pandemic on Immunosuppressed Patients: Cancer, HIV, and Solid Organ Transplant Recipients. AIDS Rev 2020;22:151-7. doi:10.24875/AIDSRev.20000052

11 Madan A, Siglin J, Khan A. Comprehensive review of implications of COVID-19 on clinical outcomes of cancer patients and management of solid tumors during the pandemic. Cancer Med 2020;9:9205-18. doi:10.1002/cam4.3534

12 Pereira MR, Mohan S, Cohen DJ, et al. COVID-19 in solid organ transplant recipients: Initial report from the US epicenter. Am J Transplant 2020;20:1800-8. doi:10.1111/ajt.15941

13 Curtis JR, Johnson SR, Anthony DD, et al. American College of Rheumatology Guidance for COVID-19 Vaccination in Patients With Rheumatic and Musculoskeletal Diseases: Version 3. Arthritis Rheumatol 2021:73:e60-75.

14 Ssentongo P, Heilbrunn ES, Ssentongo AE, et al. Epidemiology and outcomes of COVID-19 in HIV-infected individuals: a systematic review and meta-analysis. Sci Rep 2021;11:6283. doi:10.1038/ s41598-021-85359-3

15 Chong PP, Handler L, Weber DJ. A Systematic Review of Safety and Immunogenicity of Influenza Vaccination Strategies in Solid Organ Transplant Recipients. Clin Infect Dis 2018;66:1802-11. doi:10.1093/cid/cix1081

16 Westra J, van Assen S, Wilting KR, et al. Rituximab impairs immunoglobulin (Ig)M and IgG (subclass) responses after influenza vaccination in rheumatoid arthritis patients. Clin Exp Immunol 2014;178:40-7. doi:10.1111/cei.12390

17 Beck CR, McKenzie BC, Hashim AB, Harris RC, Nguyen-Van-Tam IS, University of Nottingham Influenza and the ImmunoCompromised (UNIIC) Study Group. Influenza vaccination for immunocompromised patients: systematic review and meta-analysis by etiology. I Infect Dis 2012:206:1250-9. doi:10.1093/infdis/jis487

18 Page MJ, McKenzie JE, Bossuyt PM, et al. The PRISMA 2020 statement: an updated guideline for reporting systematic reviews. BM/ 2021:372:n71. doi:10.1136/bmj.n71

19 McInnes IB, Gravallese EM. Immune-mediated inflammatory disease therapeutics: past, present and future. Nat Rev Immunol 2021:21:680-6. doi:10.1038/s41577-021-00603-1

20 Sterne JA, Hernán MA, Reeves BC, et al. ROBINS-I: a tool for assessing risk of bias in non-randomised studies of interventions. BMJ 2016;355:i4919 doi:10.1136/bmj.i4919

21 Sterne JAC, Savović J, Page MJ, et al. RoB 2: a revised tool for assessing risk of bias in randomised trials. BMJ 2019;366:14898. doi:10.1136/bmj.l4898

22 Higgins JP, Thompson SG. Quantifying heterogeneity in a metaanalysis. Stat Med 2002;21:1539-58. doi:10.1002/sim.1186

23 Duval S, Tweedie R. Trim and fill: A simple funnel-plot-based method of testing and adjusting for publication bias in meta-analysis. Biometrics 2000:56:455-63. doi:10.1111/j.0006-341X.2000.00455.x 
24 Peters JL, Sutton AJ, Jones DR, Abrams KR, Rushton L. Performance of the trim and fill method in the presence of publication bias and between-study heterogeneity. Stat Med 2007;26:4544-62. doi:10.1002/sim.2889

25 Egger M, Davey Smith G, Schneider M, Minder C. Bias in metaanalysis detected by a simple, graphical test. BMJ 1997;315:629-34. doi:10.1136/bmj.315.7109.629

26 Guyatt GH, Oxman AD, Vist GE, et al, GRADE Working Group. GRADE: an emerging consensus on rating quality of evidence and strength of recommendations. BMJ 2008;336:924-6. doi:10.1136/ bmj.39489.470347.AD

27 Rahav G, Lustig Y, Lavee J, et al. BNT162b2 mRNA COVID-19 vaccination in immunocompromised patients: A prospective cohort study. EClinicalMedicine 2021;41:101158. doi:10.1016/j. eclinm.2021.101158

28 Monin L, Laing AG, Muñoz-Ruiz M, et al. Safety and immunogenicity of one versus two doses of the COVID-19 vaccine BNT162b2 for patients with cancer: interim analysis of a prospective observational study. Lancet Oncol 2021;22:765-78. doi:10.1016/S14702045(21)00213-8

29 Boekel L, Steenhuis M, Hooijberg F, et al. Antibody development after COVID-19 vaccination in patients with autoimmune diseases in the Netherlands: a substudy of data from two prospective cohort studies. Lancet Rheumatol 2021;3:e778-88. doi:10.1016/S26659913(21)00222-8

30 Terpos E, Zagouri F, Liontos M, et al. Low titers of SARS-CoV-2 neutralizing antibodies after first vaccination dose in cancer patients receiving checkpoint inhibitors. J Hematol Oncol 2021;14:86. doi:10.1186/s13045-021-01099-x

31 Palich R, Veyri M, Marot S, et al. Weak immunogenicity after a single dose of SARS-CoV-2 mRNA vaccine in treated cancer patients. Ann Oncol 2021;32:1051-3. doi:10.1016/j.annonc.2021.04.020

32 Palich R, Veyri M, Vozy A, et al. High seroconversion rate but low antibody titers after two injections of BNT162b2 (Pfizer-BioNTech) vaccine in patients treated with chemotherapy for solid cancers. Ann Oncol 2021;32:1294-5. doi:10.1016/j.annonc.2021.06.018

33 Massarweh A, Eliakim-Raz N, Stemmer A, et al. Evaluation of Seropositivity Following BNT162b2 Messenger RNA Vaccination for SARS-CoV-2 in Patients Undergoing Treatment for Cancer. JAMA Oncol 2021;7:1133-40. doi:10.1001/jamaoncol.2021.2155

34 Eliakim-Raz N, Massarweh A, Stemmer A, Stemmer SM. Durability of Response to SARS-CoV-2 BNT162b2 Vaccination in Patients on Active Anticancer Treatment. JAMA Oncol 2021:7:1716-8. doi:10.1001/jamaoncol.2021.4390

35 Goshen-Lago T, Waldhorn I, Holland R, et al. Serologic Status and Toxic Effects of the SARS-CoV-2 BNT162b2 Vaccine in Patients Undergoing Treatment for Cancer. JAMA Oncol 2021;7:1507-13. doi:10.1001/jamaoncol.2021.2675

36 Linardou H, Spanakis N, Koliou GA et al. Responses to SARS-CoV-2 Vaccination in Patients with Cancer (ReCOVer Study): A Prospective Cohort Study of the Hellenic Cooperative Oncology Group. Cancers (Basel) 2021;13:4621. doi:10.3390/cancers13184621

37 Liontos M, Terpos E, Markellos C, et al. Immunological Response to COVID-19 Vaccination in Ovarian Cancer Patients Receiving PARP Inhibitors. Vaccines (Basel) 2021;9:1148. doi:10.3390/ vaccines9101148

38 Liontos M, Terpos E, Kunadis E, et al. Treatment with abiraterone or enzalutamide does not impair immunological response to COVID-19 vaccination in prostate cancer patients. Prostate Cancer Prostatic Dis 2021; published online 9 October. doi:10.1038/s41391-02100455-9

39 Agbarya A, Sarel I, Ziv-Baran T, et al. Efficacy of the mRNA-Based BNT162b2 COVID-19 Vaccine in Patients with Solid Malignancies Treated with Anti-Neoplastic Drugs. Cancers (Basel) 2021;13:4191. doi:10.3390/cancers13164191

40 Di Noia V, Pimpinelli F, Renna D, et al. Immunogenicity and Safety of COVID-19 Vaccine BNT162b2 for Patients with Solid Cancer: A Large Cohort Prospective Study from a Single Institution. Clin Cancer Res 2021;27:6815-23. doi:10.1158/1078-0432.CCR-21-2439

41 Grinshpun A, Rottenberg Y, Ben-Dov IZ, Djian E, Wolf DG, Kadouri L. Serologic response to COVID-19 infection and/or vaccine in cancer patients on active treatment. ESMO Open 2021;6:100283. doi:10.1016/i.esmoop.2021.100283

42 Shmueli ES, Itay A, Margalit O, et al. Efficacy and safety of BNT162b2 vaccination in patients with solid cancer receiving anticancer therapy - a single centre prospective study. Eur J Cancer 2021;157:124-31. doi:10.1016/j.ejca.2021.08.007

43 Shroff RT, Chalasani P, Wei R, et al. Immune responses to two and three doses of the BNT162b2 mRNA vaccine in adults with solid tumors. Nat Med 2021;27:2002-11. doi:10.1038/s41591-021-01542-z

44 Zagouri F, Terpos E, Fiste O, et al. SARS-CoV-2 neutralizing antibodies after first vaccination dose in breast cancer patients receiving CDK4/6 inhibitors. Breast 2021;60:58-61. doi:10.1016/j. breast.2021.08.017
45 Herishanu Y, Avivi I, Aharon A, et al. Efficacy of the BNT162b2 mRNA COVID-19 vaccine in patients with chronic lymphocytic leukemia. Blood 2021:137:3165-73 doi:10.1182/blood 2021011568

46 Pimpinelli F, Marchesi F, Piaggio G, et al. Fifth-week immunogenicity and safety of anti-SARS-CoV-2 BNT162b2 vaccine in patients with multiple myeloma and myeloproliferative malignancies on active treatment: preliminary data from a single institution. J Hematol Oncol 2021;14:81. doi:10.1186/s13045-021-01090-6

47 Herzog Tzarfati K, Gutwein O, Apel A, et al. BNT162b2 COVID-19 vaccine is significantly less effective in patients with hematologic malignancies. Am J Hematol 2021;96:1195-203. doi:10.1002/ ajh.26284

48 Malard F, Gaugler B, Gozlan J, et al. Weak immunogenicity of SARS$\mathrm{CoV}-2$ vaccine in patients with hematologic malignancies. Blood Cancer / 2021;11:142. doi:10.1038/s41408-021-00534-z

49 Parry H, Mcllroy G, Bruton R, et al. Antibody responses after first and second Covid-19 vaccination in patients with chronic lymphocytic leukaemia. Blood Cancer J 2021;11:136. doi:10.1038/s41408021-00528-x

50 Chowdhury O, Bruguier H, Mallett G, et al. Impaired antibody response to COVID-19 vaccination in patients with chronic myeloid neoplasms. BrJ Haematol 2021;194:1010-5. doi:10.1111/ bjh.17644

51 Gavriatopoulou M, Terpos E, Kastritis E, et al. Low neutralizing antibody responses in WM, CLL and NHL patients after the first dose of the BNT162b2 and AZD1222 vaccine. Clin Exp Med 2021; published online 20 July. doi:10.1007/s10238-021-00746-4

52 Gavriatopoulou M, Terpos E, Ntanasis-Stathopoulos I, et al. Poor neutralizing antibody responses in 106 patients with WM afte vaccination against SARS-CoV-2: a prospective study. Blood Adv 2021;5:4398-405. doi:10.1182/bloodadvances.2021005444

53 Lindemann M, Klisanin V, Thümmler L, et al. Humoral and Cellular Vaccination Responses against SARS-CoV-2 in Hematopoietic Stem Cell Transplant Recipients. Vaccines (Basel) 2021;9:1075. doi:10.3390/vaccines9101075

54 Marasco V, Carniti C, Guidetti A, et al. T-cell immune response after mRNA SARS-CoV-2 vaccines is frequently detected also in the absence of seroconversion in patients with lymphoid malignancies. BrJ Haematol 2022;196:548-58.

55 Terpos E, Gavriatopoulou M, Fotiou D, et al. Poor Neutralizing Antibody Responses in 132 Patients with CLL, NHL and HL after Vaccination against SARS-CoV-2: A Prospective Study. Cancers (Basel) 2021:13:4480 doi:10.3390/cancers13174480

56 Shem-Tov N, Yerushalmi R, Danylesko I, et al. Immunogenicity and safety of the BNT162b2 mRNA COVID-19 vaccine in haematopoietic stem cell transplantation recipients. Br J Haematol 2021; published online 28 October. doi:10.1111/bjh.17918

57 Sherman AC, Desjardins M, Cheng CA, et al. SARS-CoV-2 mRNA Vaccines in Allogeneic Hematopoietic Stem Cell Transplant Recipients: Immunogenicity and Reactogenicity. Clin Infect Dis 2021; ciab930. doi:10.1093/cid/ciab930

58 Peeters M, Verbruggen L, Teuwen L, et al. Reduced humoral immune response after BNT162b2 coronavirus disease 2019 messenger RNA vaccination in cancer patients under antineoplastic treatment. ESMO Open 2021;6:100274. doi:10.1016/j.esmoop.2021.100274

59 Lim SH, Campbell N, Johnson M, et al. Antibody responses after SARS-CoV-2 vaccination in patients with lymphoma. Lancet Haematol 2021;8:e542-4. doi:10.1016/S2352-3026(21)00199-X

60 Ghione P, Gu JJ, Attwood K, et al. Impaired humoral responses to COVID-19 vaccination in patients with lymphoma receiving B-cell-directed therapies. Blood 2021;138:811-4. doi:10.1182/ blood.2021012443

61 Stampfer SD, Goldwater MS, Jew S, et al. Response to mRNA vaccination for COVID-19 among patients with multiple myeloma. Leukemia 2021;35:3534-41. doi:10.1038/s41375-021-01354-7

62 Canti L, Humblet-Baron S, Desombere I, et al. Predictors of neutralizing antibody response to BNT162b2 vaccination in allogeneic hematopoietic stem cell transplant recipients. J Hematol Oncol 2021;14:174. doi:10.1186/s13045-021-01190-3

63 Yi SG, Knight RJ, Graviss EA, et al. Kidney Transplant Recipients Rarely Show an Early Antibody Response Following the First COVID-19 Vaccine Administration. Transplantation 2021;105:e72-3. doi:10.1097/TP.0000000000003764

64 Grupper A, Katchman E, Ben-Yehoyada M, et al. Kidney transplant recipients vaccinated before transplantation maintain superior humoral response to SARS-CoV-2 vaccine. Clin Transplant 2021:35:e14478 doi:10.1111/ctr.14478

65 Grupper A, Rabinowich L, Schwartz D, et al. Reduced humoral response to mRNA SARS-CoV-2 BNT162b2 vaccine in kidney transplant recipients without prior exposure to the virus. Am J Transplant 2021;21:2719-26. doi:10.1111/ajt.16615

66 Rabinowich L, Grupper A, Baruch R, et al. Low immunogenicity to SARS-CoV-2 vaccination among liver transplant recipients. Hepatol 2021;75:435-8. doi:10.1016/j.jhep.2021.04.020 
67 Mazzola A, Todesco E, Drouin S, et al. Poor Antibody Response after Two Doses of SARS-CoV-2 vaccine in Transplant Recipients. Clin Infect Dis 2021;ciab580. doi:10.1093/cid/ciab580

68 Sattler A, Schrezenmeier E, Weber UA, et al. Impaired humoral and cellular immunity after SARS-CoV-2 BNT162b2 (tozinameran) prime-boost vaccination in kidney transplant recipients. J Clin Invest 2021;131:150175. doi:10.1172/JCl150175

69 Marinaki S, Adamopoulos S, Degiannis D, et al. Immunogenicity of SARS-CoV-2 BNT162b2 vaccine in solid organ transplant recipients. Am J Transplant 2021;21:2913-5. doi:10.1111/ajt.16607

70 Miele M, Busà R, Russelli G, et al. Impaired anti-SARS-CoV-2 humoral and cellular immune response induced by Pfizer-BioNTech BNT162b2 mRNA vaccine in solid organ transplanted patients. Am J Transplant 2021;21:2919-21. doi:10.1111/ajt.16702

71 Peled Y, Ram E, Lavee J, et al. BNT162b2 vaccination in heart transplant recipients: Clinical experience and antibody response. J Heart Lung Transplant 2021;40:759-62. doi:10.1016/j. healun.2021.04.003

72 Hod T, Ben-David A, Olmer L, et al. Humoral Response of Renal Transplant Recipients to the BNT162b2 SARS-CoV-2 mRNA Vaccine Using Both RBD IgG and Neutralizing Antibodies. Transplantation 2021;105:e234-43. doi:10.1097/ TP.0000000000003889

73 Narasimhan M, Mahimainathan L, Clark AE, et al. Serological Response in Lung Transplant Recipients after Two Doses of SARSCoV-2 mRNA Vaccines. Vaccines (Basel) 2021;9:708. doi:10.3390/ vaccines 9070708

74 Rashidi-Alavijeh J, Frey A, Passenberg M, et al. Humoral Response to SARS-Cov-2 Vaccination in Liver Transplant Recipients-A SingleCenter Experience. Vaccines (Basel) 2021;9:738. doi:10.3390 vaccines 9070738

75 Schramm R, Costard-Jäckle A, Rivinius R, et al. Poor humoral and T-cell response to two-dose SARS-CoV-2 messenger RNA vaccine BNT162b2 in cardiothoracic transplant recipients. Clin Res Cardiol 2021;110:1142-9. doi:10.1007/s00392-021-01880-5

76 Stumpf J, Siepmann T, Lindner T, et al. Humoral and cellular immunity to SARS-CoV-2 vaccination in renal transplant versus dialysis patients: A prospective, multicenter observational study using mRNA-1273 or BNT162b2 mRNA vaccine. Lancet Reg Health Eur 2021:100178.

77 Rincon-Arevalo H, Choi M, Stefanski AL, et al. Impaired humoral immunity to SARS-CoV-2 BNT162b2 vaccine in kidney transplant recipients and dialysis patients. Sci Immunol 2021;6:eabj1031. doi:10.1126/sciimmunol.abj1031

78 Danthu C, Hantz S, Dahlem A, et al. Humoral Response after SARSCoV-2 mRNA Vaccination in a Cohort of Hemodialysis Patients and Kidney Transplant Recipients. J Am Soc Nephrol 2021;32:2153-8. doi:10.1681/ASN.2021040490

79 Korth J, Jahn M, Dorsch O, et al. Impaired Humoral Response in Rena Transplant Recipients to SARS-CoV-2 Vaccination with BNT162b2 (Pfizer-BioNTech). Viruses 2021;13:756. doi:10.3390/v13050756

80 Prendecki M, Clarke C, Edwards $\mathrm{H}$, et al. Humoral and T-cell responses to SARS-CoV-2 vaccination in patients receiving immunosuppression. Ann Rheum Dis 2021;80:1322-9. doi:10.1136/annrheumdis-2021-220626

81 Ruether DF, Schaub GM, Duengelhoef PM, et al. SARS-CoV2-specific Humoral and T-cell Immune Response After Second Vaccination in Liver Cirrhosis and Transplant Patients. Clin Gastroenterol Hepatol 2022;20:162-172.e9.

82 Bruminhent J, Setthaudom C, Chaumdee P, et al, Ramathibodi Transplant Infectious Diseases (RTID) Study Group. SARS-CoV2-specific humoral and cell-mediated immune responses after immunization with inactivated COVID-19 vaccine in kidney transplant recipients (CVIM 1 study). Am J Transplant 2021; published online 17 October. doi:10.1111/ajt.16867

83 Crespo M, Barrilado-Jackson A, Padilla E, et al, Mariscovid Research Group. Negative immune responses to two-dose mRNA COVID-19 vaccines in renal allograft recipients assessed with simple antibody and interferon gamma release assay cellular monitoring. Am J Transplant 2021; published online 22 September. doi:10.1111/ ajt. 16854

84 D’Offizi G, Agrati C, Visco-Comandini U, et al. Coordinated cellular and humoral immune responses after two-dose SARS-CoV2 mRNA vaccination in liver transplant recipients. Liver Int 2022;42:180-6.

85 Dębska-Ślizień A, Ślizień Z, Muchlado M, et al. Predictors of Humoral Response to mRNA COVID19 Vaccines in Kidney Transplant Recipients: A Longitudinal Study-The COViNEPH Project. Vaccines (Basel) 2021;9:1165. doi:10.3390/vaccines9101165

86 Kantauskaite M, Müller L, Kolb T, et al. Intensity of mycophenolate mofetil treatment is associated with an impaired immune response to SARS-CoV-2 vaccination in kidney transplant recipients. Am J Transplant 2021; published online 22 September. doi:10.1111/ ajt.16851
87 Schmidt T, Klemis V, Schub D, et al. Cellular immunity predominates over humoral immunity after homologous and heterologous mRNA and vector-based COVID-19 vaccine regimens in solid organ transplant recipients. Am J Transplant 2021;21:3990-4002. doi:10.1111/ajt.16818

88 Furer V, Eviatar T, Zisman D, et al. Immunogenicity and safety of the BNT162b2 mRNA COVID-19 vaccine in adult patients with autoimmune inflammatory rheumatic diseases and in the general population: a multicentre study. Ann Rheum Dis 2021;80:1330-8. doi:10.1136/annrheumdis-2021-220647

89 Rubbert-Roth A, Vuilleumier N, Ludewig B, Schmiedeberg K, Haller C, von Kempis J. Anti-SARS-CoV-2 mRNA vaccine in patients with rheumatoid arthritis. Lancet Rheumatol 2021;3:e470-2. doi:10.1016/S2665-9913(21)00186-7

90 Deepak P, Kim W, Paley MA, et al. Effect of Immunosuppression on the Immunogenicity of mRNA Vaccines to SARS-CoV-2: A Prospective Cohort Study. Ann Intern Med 2021;174:1572-85. doi:10.7326/ M21-1757

91 Achiron A, Mandel M, Dreyer-Alster S, et al. Humoral immune response to COVID-19 mRNA vaccine in patients with multiple sclerosis treated with high-efficacy disease-modifying therapies. Ther Adv Neurol Disord 2021;14:17562864211012835. doi:10.1177/17562864211012835

92 Mahil SK, Bechman K, Raharja A, et al. The effect of methotrexate and targeted immunosuppression on humoral and cellular immune responses to the COVID-19 vaccine BNT162b2: a cohort study. Lancet Rheumatol 2021;3:e627-37. doi:10.1016/S26659913(21)00212-5

93 Medeiros-Ribeiro AC, Aikawa NE, Saad CGS, et al. Immunogenicity and safety of the CoronaVac inactivated vaccine in patients with autoimmune rheumatic diseases: a phase 4 trial. Nat Med 2021;27:1744-51. doi:10.1038/s41591-021-01469-5

94 Reuken PA, Andreas N, Grunert PC, Glöckner S, Kamradt T, Stallmach A. T cell response after SARS-CoV-2 vaccination in immunocompromised patients with inflammatory bowel disease. J Crohns Colitis 2021;jiab147. doi:10.1093/ecco-jcc/jiab147

95 Seyahi E, Bakhdiyarli G, Oztas M, et al. Antibody response to inactivated COVID-19 vaccine (CoronaVac) in immune-mediated diseases: a controlled study among hospital workers and elderly. Rheumatol Int 2021:41:1429-40 doi:10.1007/s00296-021-04910-7

96 Haberman RH, Herati R, Simon D, et al. Methotrexate hampers immunogenicity to BNT162b2 mRNA COVID-19 vaccine in immunemediated inflammatory disease. Ann Rheum Dis 2021;80:1339-44 doi:10.1136/annrheumdis-2021-220597

97 Simon D, Tascilar K, Fagni F, et al. SARS-CoV-2 vaccination responses in untreated, conventionally treated and anticytokine-treated patients with immune-mediated inflammatory diseases. Ann Rheum Dis 2021;80:1312-6. doi:10.1136/annrheumdis-2021-220461

98 Mrak D, Tobudic S, Koblischke M, et al. SARS-CoV-2 vaccination in rituximab-treated patients: $B$ cells promote humoral immune responses in the presence of T-cell-mediated immunity. Ann Rheum Dis 2021;80:1345-50. doi:10.1136/annrheumdis-2021-220781

99 Achiron A, Mandel M, Dreyer-Alster S, et al. Humoral immune response in multiple sclerosis patients following PfizerBNT162b2 COVID19 vaccination: Up to 6 months cross-sectional study. / Neuroimmunol 2021;361:577746. doi:10.1016/j. jneuroim.2021.577746

100 Ali A, Dwyer D, Wu Q, et al. Characterization of humoral response to COVID mRNA vaccines in multiple sclerosis patients on disease modifying therapies. Vaccine 2021;39:6111-6. doi:10.1016/j. vaccine.2021.08.078

101 Shinjo SK, de Souza FHC, Borges IBP, et al. Systemic autoimmune myopathies: A prospective phase 4 controlled trial of an inactivated virus vaccine against SARS-CoV-2. Rheumatology (Oxford) 2021; published online 19 October. doi:10.1093/rheumatology/keab773

102 Levy I, Wieder-Finesod A, Litchevsky V, et al. Immunogenicity and safety of the BNT162b2 mRNA COVID-19 vaccine in people living with HIV-1. Clin Microbiol Infect 2021;27:1851-5. doi:10.1016/j.cmi.2021.07.031

103 Madhi SA, Koen AL, Izu A, et al, Wits VIDA COVID team. Safety and immunogenicity of the ChAdOx1 nCoV-19 (AZD1222) vaccine against SARS-CoV-2 in people living with and without HIV in South Africa: an interim analysis of a randomised, double-blind, placebo-controlled, phase 1B/2A trial. Lancet HIV 2021;8:e568-80. doi:10.1016/S2352-3018(21)00157-0

104 Woldemeskel BA, Karaba AH, Garliss CC, et al. The BNT162b2 mRNA Vaccine Elicits Robust Humoral and Cellular Immune Responses in People Living with HIV. Clin Infect Dis 2021;ciab648. doi:10.1093/ cid/ciab648

105 Prendecki M, Thomson T, Clarke CL, et al, Imperial Renal COVID-19 vaccine study group in collaboration with the OCTAVE Study Consortium. Immunological responses to SARS-CoV-2 vaccines in kidney transplant recipients. Lancet 2021;398:1482-4. doi:10.1016/S0140-6736(21)02096-1 
106 Bitoun S, Henry J, Vauloup-Fellous C, et al. Response to COVID-19 mRNA vaccination in multiple myeloma is conserved but impaired compared to controls. J Hematol Oncol 2021;14:166. doi:10.1186 s13045-021-01183-2

107 Wong SY, Dixon R, Martinez Pazos V, Gnjatic S, Colombel JF, Cadwell K, et al, ICARUS-IBD Working Group. Serologic Response to Messenger RNA Coronavirus Disease 2019 Vaccines in Inflammatory Bowel Disease Patients Receiving Biologic Therapies. Gastroenterology 2021;161:715-718.e4. doi:10.1053/j. gastro.2021.04.025

108 Barrière J, Chamorey E, Adjtoutah Z, et al. Impaired immunogenicity of BNT162b2 anti-SARS-CoV-2 vaccine in patients treated for solid tumors. Ann Oncol 2021:32:1053-5. doi:10.1016/j. annonc.2021.04.019

109 lacono D, Cerbone L, Palombi L, et al. Serological response to COVID-19 vaccination in patients with cancer older than 80 years. I Geriatr Oncol 2021;12:1253-5. doi:10.1016/j.jgo.2021.06.002

110 Izmirly PM, Kim MY, Samanovic M, et al. Evaluation of Immune Response and Disease Status in SLE Patients Following SARS-CoV-2 Vaccination. Arthritis Rheumatol 2022;74:284-94. doi:10.1002/art.41937

111 Apostolidis SA, Kakara M, Painter MM, et al. Cellular and humoral immune responses following SARS-CoV-2 mRNA vaccination in patients with multiple sclerosis on anti-CD20 therapy. Nat Med 2021;27:1990-2001. doi:10.1038/s41591-021-01507-2

112 Jedicke N, Stankov MV, Cossmann A, et al. Humoral immune response following prime and boost BNT162b2 vaccination in people living with HIV on antiretroviral therapy. HIV Med 2021; published online 2 November. doi:10.1111/hiv.13202

113 Geisen UM, Berner DK, Tran F, et al. Immunogenicity and safety of anti-SARS-CoV-2 mRNA vaccines in patients with chronic inflammatory conditions and immunosuppressive therapy in a monocentric cohort. Ann Rheum Dis 2021;80:1306-11. doi:10.1136/annrheumdis-2021-220272

114 Greenberger LM, Saltzman LA, Senefeld JW, Johnson PW, DeGennaro LJ, Nichols GL. Anti-spike antibody response to SARS-CoV-2 booster vaccination in patients with $B$ cell-derived hematologic malignancies. Cancer Cell 2021;39:1297-9. doi:10.1016/j.ccell.2021.09.001

115 Chavarot N, Morel A, Leruez-Ville M, et al. Weak antibody response to three doses of $m$ RNA vaccine in kidney transplant recipients treated with belatacept. Am J Transplant 2021;21:4043-51. doi:10.1111/ ajt.16814

116 Benotmane I, Gautier G, Perrin P, et al. Antibody Response After a Third Dose of the mRNA-1273 SARS-CoV-2 Vaccine in Kidney Transplant Recipients With Minimal Serologic Response to 2 Doses. JAMA 2021;326:1063-6.5. doi:10.1001/jama.2021.12339

117 Del Bello A, Abravanel F, Marion O, et al. Efficiency of a boost with a third dose of anti-SARS-CoV-2 messenger RNA-based vaccines in solid organ transplant recipients. Am J Transplant 2022;22:322-3.

118 Peled Y, Ram E, Lavee J, et al. Third dose of the BNT162 b2 vaccine in heart transplant recipients: Immunogenicity and clinical experience. J Heart Lung Transplant 2021;S1053-2498(21)02481-5.

119 Westhoff TH, Seibert FS, Anft M, et al. A third vaccine dose substantially improves humoral and cellular SARS-CoV-2 immunity in renal transplant recipients with primary humoral nonresponse. Kidney Int 2021;100:1135-6. doi:10.1016/j.kint.2021.09.001

120 Bertrand D, Hamzaoui M, Lemée V, et al. Antibody and T-cell response to $a$ third dose of SARS-CoV-2 mRNA BNT162b2 vaccine in kidney transplant recipients. Kidney Int 2021;100:1337-40. doi:10.1016/j. kint.2021.09.014

121 Schrezenmeier E, Rincon-Arevalo H. Stefanski A, et al L. B and T Cell Responses after a Third Dose of SARS-CoV-2 Vaccine in Kidney Transplant Recipients. J Am Soc Nephrol 2021;ASN.2021070966. doi:10.1681/ASN.2021070966

122 Hall VG, Ferreira VH, Ku T, et al. Randomized Trial of a Third Dose of mRNA-1273 Vaccine in Transplant Recipients. N Engl J Med 2021;385:1244-6. doi:10.1056/NEJMc2111462

123 Schmiedeberg K, Vuilleumier N, Pagano S, et al. Efficacy and tolerability of a third dose of an mRNA anti-SARS-CoV-2 vaccine in patients with rheumatoid arthritis with absent or minimal serological response to two previous doses. Lancet Rheumatol 2022:4:e11-3.

124 Doria-Rose N, Suthar MS, Makowski M, et al, mRNA-1273 Study Group. Antibody Persistence through 6 Months after the Second Dose of mRNA-1273 Vaccine for Covid-19. N Engl J Med 2021;384:225961. doi:10.1056/NEJMc2103916
125 Livingston EH. Necessity of 2 Doses of the Pfizer and Moderna COVID-19 Vaccines. JAMA 2021;325:898. doi:10.1001/ jama.2021.1375

126 Silva-Cayetano A, Foster WS, Innocentin S, et al. A booster dose enhances immunogenicity of the COVID-19 vaccine candidate ChAdOx1 nCoV-19 in aged mice. Med (N Y) 2021;2:243-262.e8. doi:10.1016/j.medj.2020.12.006

127 Pregliasco F, Grilli G, Andreassi A, et al. Immune response to a booster dose of enhanced potency inactivated polio vaccine administered in association with HBV vaccine in adolescents. Vaccine 1996;14:267-9. doi:10.1016/0264-410X(95)00209-J

128 US Food and Drug Administration. Coronavirus (COVID-19) Update: FDA Authorizes Additional Vaccine Dose for Certain Immunocompromised Individuals. FDA, 2021 [cited 27 September 2021]. www.fda.gov/news-events/press-announcements/ coronavirus-covid-19-update-fda-authorizes-additional-vaccinedose-certain-immunocompromised.

129 Wise J. Covid-19: UK will offer third vaccine dose to severely immunosuppressed people. BM/ 2021;374:n2160. doi:10.1136/ bmj.n2160

130 American Society of Transplantation, International Society for Heart and Lung Transplantation. Joint Statement about Vaccine Efficacy in Organ Transplant Recipients. 2021. www.myast.org/ sites/default/files/ast\%20ishlt\%20guidance\%20vaccine $\% 20$ 08132021FINAL\%20DRAFT2.pdf

131 Kennedy NA, Lin S, Goodhand IR, et al, Contributors to the CLARITY IBD study. Infliximab is associated with attenuated immunogenicity to BNT162b2 and ChAdOx1 nCoV-19 SARS-CoV-2 vaccines in patients with IBD. Gut 2021;70:1884-93. doi:10.1136/gutjnl-2021-324789

132 Ligumsky H, Safadi E, Etan T, et al. Immunogenicity and Safety of the BNT162b2 mRNA COVID-19 Vaccine Among Actively Treated Cancer Patients. J Natl Cancer Inst 2021;djab174. doi:10.1093/jnci/ djab174

133 Jin P, Li J, Pan H, Wu Y, Zhu F. Immunological surrogate endpoints of COVID-2019 vaccines: the evidence we have versus the evidence we need. Signal Transduct Target Ther 2021;6:48. doi:10.1038/ s41392-021-00481-y

134 Khoury DS, Cromer D, Reynaldi A, et al. Neutralizing antibody levels are highly predictive of immune protection from symptomatic SARSCoV-2 infection. Nat Med 2021:27:1205-11. doi:10.1038/s41591 021-01377-8

135 Earle KA, Ambrosino DM, Fiore-Gartland A, et al. Evidence for antibody as a protective correlate for COVID-19 vaccines. Vaccine 2021;39:4423-8. doi:10.1016/j.vaccine.2021.05.063

136 Garcia-Beltran WF, Lam EC, Astudillo MG, et al. COVID-19-neutralizing antibodies predict disease severity and survival. Cell 2021;184:476488.e11. doi:10.1016/i.cell.2020.12.015

137 Anema A, Mills E, Montaner J, Brownstein JS, Cooper C. Efficacy of influenza vaccination in HIV-positive patients: a systematic review and meta-analysis. HIV Med 2008;9:57-61. doi:10.1111/j.14681293.2008.00515.X

138 Catherine FX, Piroth L. Hepatitis B virus vaccination in HIV-infected people: A review. Hum Vaccin Immunother 2017:13:1-10. doi:10.10 80/21645515.2016.1277844

139 Lee JH, Hong S, Im JH, Lee JS, Baek JH, Kwon HY. Systematic review and meta-analysis of immune response of double dose of hepatitis B vaccination in HIV-infected patients. Vaccine 2020;38:3995-4000. doi:10.1016/j.vaccine.2020.04.022

140 Roifman CM, Vong L. COVID-19 vaccination for patients with primary immunodeficiency. LymphoSign Journal. 2021:8:37-45. doi:10.14785/lymphosign-2021-0020

141 Hagin D, Freund T, Navon M, et al. Immunogenicity of Pfizer-BioNTech COVID-19 vaccine in patients with inborn errors of immunity. J Allergy Clin Immunol 2021;148:739-49. doi:10.1016/j.jaci.2021.05.029

142 Fan YJ, Chan KH, Hung IF. Safety and Efficacy of COVID-19 Vaccines: A Systematic Review and Meta-Analysis of Different Vaccines at Phase 3. Vaccines (Basel) 2021;9:989. doi:10.3390/vaccines9090989

143 Zbinden D, Manuel O. Influenza vaccination in immunocompromised patients: efficacy and safety. Immunotherapy 2014;6:131-9. doi:10.2217/imt.13.171

Supplementary information: additional tables 1-20 and additional figures 1-21 